Pacific

Journal of

Mathematics

THE PLURICOMPLEX GREEN FUNCTION WITH TWO POLES OF THE UNIT BALL OF $\mathbb{C}^{n}$

Dan COMAN

Volume $194 \quad$ No. 2

June 2000 


\title{
THE PLURICOMPLEX GREEN FUNCTION WITH TWO POLES OF THE UNIT BALL OF $\mathbb{C}^{n}$
}

\author{
DAN COMAN
}

\begin{abstract}
In this paper we find the formula for the pluricomplex Green function of the unit ball of $\mathbb{C}^{n}$ with two poles of equal weights. The strategy will be to show the existence of a foliation of the ball (singular at the poles) by proper smooth analytic discs passing through one or through both of the poles, such that the restriction of the pluricomplex Green function to these discs is harmonic away from the poles. This foliation is obtained by solving a suitable extremal problem, in analogy to the results of Lempert in the case of one pole for convex domains. Using the expression of the Green function along each leaf of the foliation, we construct its formula on the whole ball. We then show that this function is of class $C^{1,1}$ but not $C^{2}$.
\end{abstract}

\section{Introduction and statement of results.}

Let us recall the definition of the pluricomplex Green function and its connection to the complex Monge-Ampère operator. Let $\Omega$ be a bounded open set in $\mathbb{C}^{n}$ and let $p$ be a point in $\Omega$. A plurisubharmonic function $v$ on $\Omega$ is said to have a logarithmic pole at $p$ with weight $\nu>0$ if $v(z) \leq \nu \log \|z-p\|+c$, for some constant $c$ and for $z$ in a neighborhood of $p$. The pluricomplex Green function $g_{\Omega}(z, p)$ of $\Omega$ with pole at $p$ is defined by $g_{\Omega}(z, p)=\sup v(z)$, where the supremum is taken over the set of negative plurisubharmonic functions $v$ on $\Omega$ which have a logarithmic pole at $p$ with weight $\nu=1$. This definition, given by Klimek [K1], is in analogy to the one dimensional case, where one obtains in this way the (negative) Green function for the Laplace operator. The function $g_{\Omega}(\cdot, p)$ is negative and plurisubharmonic in $\Omega$, maximal in $\Omega \backslash\{p\}$, and it has a logarithmic pole at $p$. It is also decreasing with respect to holomorphic mappings, i.e., $g_{\Omega^{\prime}}(f(z), f(p)) \leq g_{\Omega}(z, p)$, where $\Omega^{\prime}$ is a bounded open set in $\mathbb{C}^{m}$ and $f: \Omega \rightarrow \Omega^{\prime}$ is a holomorphic mapping. It follows that $g_{\Omega}$ is biholomorphically invariant. In the case of the unit ball $B$ of $\mathbb{C}^{n}$ we have $g_{B}(z, 0)=\log \|z\|$. Since the automorphism group of $B$ is transitive we see that $g_{B}(z, p)=g_{B}(T z, 0)$, where $T \in \operatorname{Aut}(B)$ satisfies $T(p)=0$. 
If $\Omega$ is a hyperconvex domain (i.e., it has a negative plurisubharmonic exhaustion function) and if for $z \in \partial \Omega$ and $p \in \Omega$ we define $g_{\Omega}(z, p)=0$, then $g_{\Omega}: \bar{\Omega} \times \Omega \rightarrow[-\infty, 0]$ is continuous. This result was obtained by Demailly [D1]. He also showed that when $\Omega$ is hyperconvex $g_{\Omega}(\cdot, p)$ is the unique solution of the following Dirichlet problem for the complex Monge-Ampère operator: $u \in P S H(\Omega) \bigcap C(\bar{\Omega} \backslash\{p\}), u(z)-\log \|z-p\|=O(1)$ as $z \rightarrow p$, $\left(d d^{c} u\right)^{n}=\delta_{p}$ in $\Omega, u=0$ on $\partial \Omega$. Here $d=\partial+\bar{\partial}, d^{c}=\frac{1}{2 \pi i}(\partial-\bar{\partial})$, and $\delta_{p}$ is the Dirac mass at $p$. The Monge-Ampère operator $\left(d d^{c} u\right)^{n}$ acting on locally bounded plurisubharmonic functions was defined by Bedford and Taylor $[\mathbf{B T}]$ (we also refer to $[\mathbf{D 2}]$ and $[\mathbf{K 2}]$ for a detailed presentation). We recall that the definition of the Monge-Ampère operator can be extended so that it applies to plurisubharmonic functions with finitely many singularities. We refer to $[\mathbf{D 2}]$ and $[\mathbf{F S}]$ for extensions of the complex Monge-Ampère operator to suitable classes of unbounded plurisubharmonic functions.

The pluricomplex Green function with finitely many poles was introduced and studied by Lelong [L]. If $\Omega$ is a bounded open set in $\mathbb{C}^{n}$ and $A=$ $\left\{\left(p_{j}, \nu_{j}\right) \in \Omega \times(0, \infty): j=1, \ldots, k\right\}$ the pluricomplex Green function $g_{\Omega}(\cdot, A)$ of $\Omega$ with poles in $A$ is defined by $g_{\Omega}(z, A)=\sup v(z)$, where the supremum is taken over the set of negative plurisubharmonic functions $v$ on $\Omega$ which have a logarithmic pole at $p_{j}$ with weight $\nu_{j}, j=1, \ldots, k$. It is easy to see that $g_{\Omega}(\cdot, A)$ is negative and plurisubharmonic on $\Omega$, maximal on $\Omega \backslash\left\{p_{1}, \ldots, p_{k}\right\}$, and it has a logarithmic pole at each $p_{j}$ with weight $\nu_{j}$. If $\Omega$ is hyperconvex and we define $g_{\Omega}(z, A)=0$ for $z \in \partial \Omega$, then $g_{\Omega}(\cdot, A): \bar{\Omega} \rightarrow[-\infty, 0]$ is continuous and $\left(d d^{c} g_{\Omega}(\cdot, A)\right)^{n}=\sum_{j=1}^{k} \nu_{j}^{n} \delta_{p_{j}}$, as measures on $\Omega$.

We note that in general we have

$$
\sum_{j=1}^{k} \nu_{j} g_{\Omega}\left(z, p_{j}\right) \leq g_{\Omega}(z, A) \leq \min \left\{\nu_{j} g_{\Omega}\left(z, p_{j}\right): j=1, \ldots, k\right\} .
$$

In dimension one the complex Monge-Ampère operator is the same as the Laplace operator, hence it is linear; so equality holds in the first inequality of the above relation. This is however far from being the case in dimensions $n \geq$ 2 (see $[\mathbf{L}]$ ), when the complex Monge-Ampère operator is highly nonlinear.

We denote by $\Delta$ the unit disc in $\mathbb{C}$ and we consider the function $\delta_{\Omega}(z, p)=$ $\inf \{\log s\}$, where the infimum is taken over all $s \in(0,1)$ for which there is an analytic disc $f: \Delta \rightarrow \Omega$ such that $f(0)=z$ and $f(s)=p$. In the case of one pole it is well known that $g_{\Omega}(z, p) \leq \delta_{\Omega}(z, p)$, for all $z \in \Omega$ (see [K2]). It was proved by Lempert that if $\Omega$ is a bounded convex domain in $\mathbb{C}^{n}$ then $g_{\Omega}(z, p)=\delta_{\Omega}(z, p)$, for all $z \in \Omega([\mathbf{L m} \mathbf{1}],[\mathbf{L m} \mathbf{2}])$. Let now $\Omega$ be a bounded strongly convex domain in $\mathbb{C}^{n}$ with real analytic (or $C^{\infty}$ ) boundary (by strongly convex we mean that $\Omega$ has a defining function whose real Hessian is positive definite on all real tangent spaces $\left.T_{p}(\partial D), p \in \partial D\right)$. Lempert 
showed in this case that the function $g_{\Omega}(\cdot, p)$ is real analytic (respectively $\left.C^{\infty}\right)$ on $\Omega \backslash\{p\}$ and that for each $z \in \Omega \backslash\{p\}$ there is a unique properly embedded analytic disc $f_{z}: \Delta \rightarrow \Omega$ such that $f_{z}(0)=z, f_{z}(t)=p$ for a unique $t>0$, and the function $\zeta \in \Delta \rightarrow g_{\Omega}\left(f_{z}(\zeta), p\right)$ is harmonic in $\Delta \backslash\{t\}$. We have in fact $g_{\Omega}\left(f_{z}(\zeta), p\right)=\log \left|\frac{\zeta-t}{1-t \zeta}\right|, \zeta \in \Delta$. Moreover, the curves $f_{z}(\Delta)$ foliate $\Omega \backslash\{p\}$. We recall that in the case of the ball $B$ the foliation corresponding to $g_{B}(\cdot, p)$ consists of the complex lines through $p$.

So far very little is known about the "structure" of the pluricomplex Green function with several poles. A natural question to ask is what are in this case the analogues of Lempert's results mentioned above. In particular, if $\Omega$ is a smoothly bounded strongly convex domain does there exist a foliation of $\Omega \backslash\{p, q\}$ by analytic discs on which the pluricomplex Green function with poles at $p$ and $q$ is harmonic? Moreover, what regularity does this function have?

In the present paper we compute the pluricomplex Green function of the unit ball of $\mathbb{C}^{n}$ with two poles, and we provide answers to the above questions in this case. We now state our results.

Let $\Omega$ be a bounded domain in $\mathbb{C}^{n}$ and let $A=\left\{\left(p_{1}, \nu_{1}\right), \ldots,\left(p_{k}, \nu_{k}\right)\right\} \subset$ $\Omega \times(0,+\infty)$. In analogy to the results mentioned above it is natural to consider the function

$$
\delta_{\Omega}(z, A)=\inf \left\{\nu_{1} \log \left|s_{1}\right|+\cdots+\nu_{k} \log \left|s_{k}\right|\right\},
$$

where the infimum is taken over all $s_{1}, \ldots, s_{k} \in \Delta$ for which there exists an analytic disc $f: \Delta \rightarrow \Omega$ such that $f(0)=z$ and $f\left(s_{j}\right)=p_{j}, j=1, \ldots, k$. Using this we define for $z \in \Omega$

$$
\delta_{\Omega}^{A}(z)=\min \left\{\delta_{\Omega}(z, S): S \subseteq A, S \neq \emptyset\right\} .
$$

We have the following proposition, whose proof will be postponed until the end of the paper.

Proposition 1. The function $z \rightarrow \delta_{\Omega}^{A}(z)$ is negative, it has a logarithmic pole with weight $\nu_{j}$ at each point $p_{j}, j=1, \ldots, k$, and it satisfies $g_{\Omega}(z, A) \leq$ $\delta_{\Omega}^{A}(z)$ on $\Omega$. Moreover, if $\Omega$ is taut then the function $\delta_{\Omega}^{A}: \Omega \rightarrow[-\infty, 0)$ is continuous.

Let us now specialize to our situation. We denote by $B^{n}$ the unit ball of $\mathbb{C}^{n}$ and by $g_{n}(\cdot, p, q)$ the pluricomplex Green function of $B^{n}$ with poles at $p \neq q \in B^{n}$ and with weight one at each pole. By using a suitable automorphism of $B^{n}$ we may assume without loss of generality that $p=$ $-q=(\beta, 0, \ldots, 0)$, for some $\beta \in(0,1)$ (we indicate how this can be done at the beginning of Section 2$)$. We also denote by $g_{n}(\cdot, p)$ and $g_{n}(\cdot, q)$ the pluricomplex Green functions of $B^{n}$ with poles at $p$ and $q$ respectively. The 
functions $\delta_{\Omega}$ introduced above take the following form:

$$
\delta_{n}(z, p)=\inf \left\{\log s: s \in(0,1), \exists f \in \mathcal{O}\left(\Delta, B^{n}\right), f(0)=z, f(s)=p\right\},
$$

$$
\delta_{n}(z, p, q)=\inf \{\log s+\log |t|\},
$$

where the infimum is taken over the set of $(s, t) \in(0,1) \times \Delta$ for which there exists $f \in \mathcal{O}\left(\Delta, B^{n}\right)$ satisfying $f(0)=z, f(s)=p, f(t)=q$, and

$$
\delta_{n}^{p, q}(z)=\min \left\{\delta_{n}(z, p), \delta_{n}(z, q), \delta_{n}(z, p, q)\right\} .
$$

Note that in the definition of $\delta_{n}(z, p, q)$ we used the normalization $s>0$ (compare with the definition of $\delta_{\Omega}(z, A)$ ); this can always be achieved by using a rotation of $\Delta$.

We remark that by Lemma 8 of $[\mathbf{A T 1}]$ we have in fact $\delta_{n}(z, p, q) \leq$ $\delta_{n}(z, p)$, so $\delta_{n}^{p, q}(z)=\delta_{n}(z, p, q)$ on $B^{n}$.

We now discuss the two dimensional case $n=2$. The general case $n \geq 2$ will follow easily from this one. So we assume $n=2$ and $p=-q=(\beta, 0)$, where $\beta \in(0,1)$. We divide $B^{2}$ into three regions: $\Gamma_{p}$ and $\Gamma_{q}$, which are intersections of $B^{2}$ with two closed complex cones with vertex at $p$ and $q$ respectively, and the complement of their union, $D$. They are defined as follows:

$$
\begin{aligned}
\Gamma_{p} & =\left\{z=\left(z_{1}, z_{2}\right) \in B^{2}:\left|\beta-z_{1}\right| \leq \beta\left|z_{2}\right|\right\}, \\
\Gamma_{q} & =\left\{z \in B^{2}:\left|\beta+z_{1}\right| \leq \beta\left|z_{2}\right|\right\}, \\
D & =B^{2} \backslash\left(\Gamma_{p} \cup \Gamma_{q}\right) \\
& =\left\{z \in B^{2}: \beta\left|z_{2}\right|<\min \left\{\left|\beta-z_{1}\right|,\left|\beta+z_{1}\right|\right\}\right\} .
\end{aligned}
$$

The main result of this paper is the following:

Theorem 2. The pluricomplex Green function of the unit ball of $\mathbb{C}^{2}$ with poles at $p=-q=(\beta, 0)$ is given by

$$
\begin{aligned}
g_{2}(z, p, q)= & \delta_{2}(z, p, q) \\
= & \left\{\begin{array}{l}
g_{2}(z, p)=\log \frac{\sqrt{\left|\beta-z_{1}\right|^{2}+\left(1-\beta^{2}\right)\left|z_{2}\right|^{2}}}{\left|1-\beta z_{1}\right|}, z \in \Gamma_{p}, \\
\frac{1}{2} \log \frac{\left|\beta^{2}-z_{1}^{2}\right|^{2}+\beta^{4}\left|z_{2}\right|^{4}+2\left(1-\beta^{4}\right)\left|z_{2}\right|^{2}+\sqrt{M(z)}}{2\left|1-\beta^{2} z_{1}^{2}\right|^{2}}, z \in D, \\
g_{2}(z, q)=\log \frac{\sqrt{\left|\beta+z_{1}\right|^{2}+\left(1-\beta^{2}\right)\left|z_{2}\right|^{2}}}{\left|1+\beta z_{1}\right|}, z \in \Gamma_{q},
\end{array}\right.
\end{aligned}
$$

where $M(z)=\left(\beta^{4}\left|z_{2}\right|^{4}-\left|\beta^{2}-z_{1}^{2}\right|^{2}\right)^{2}+\left.4\left(1-\beta^{4}\right)\left|z_{2}\right|^{2}\left|\beta^{2}\right| z_{2}\right|^{2}-\left.\left(\beta^{2}-z_{1}^{2}\right)\right|^{2}$. The function $g_{2}(\cdot, p, q)$ is real analytic in int $\Gamma_{p} \cup D \cup \operatorname{int} \Gamma_{q}$, it is of class $C^{1,1}$ on $B^{2} \backslash\{p, q\}$, and its first order partial derivatives extend continuously to $\partial B^{2}$. The domain $D$ is foliated by a one parameter family of complex curves $L_{\gamma}, \gamma \in \Delta$, which are given by the formula

$$
L_{\gamma}=\left\{z \in B^{2}: \gamma z_{1}^{2}=\beta^{2}\left(\gamma-z_{2}\right)\left(1-\bar{\gamma} z_{2}\right)\right\} .
$$


The leaves $L_{\gamma}$ are properly embedded submanifolds of $B^{2}$ and the restriction of $g_{2}(\cdot, p, q)$ to each $L_{\gamma}$ is harmonic away from $p$ and $q$.

In order to describe the Green function $g_{n}(\cdot, p, q), p=-q=(\beta, 0, \ldots, 0)$, for arbitrary $n$, we write $z=\left(z_{1}, z^{\prime}\right) \in \mathbb{C} \times \mathbb{C}^{n-1}$ and we consider the regions

$$
\begin{aligned}
\Gamma_{p} & =\left\{z \in B^{n}:\left|\beta-z_{1}\right| \leq \beta\left\|z^{\prime}\right\|\right\}, \\
\Gamma_{q} & =\left\{z \in B^{n}:\left|\beta+z_{1}\right| \leq \beta\left\|z^{\prime}\right\|\right\}, \\
D & =B^{n} \backslash\left(\Gamma_{p} \cup \Gamma_{q}\right)=\left\{z \in B^{n}: \beta\left\|z^{\prime}\right\|<\min \left\{\left|\beta-z_{1}\right|,\left|\beta+z_{1}\right|\right\}\right\} .
\end{aligned}
$$

For $u=\left(0, u^{\prime}\right) \in \partial B^{n}$ we let $V_{u}=\mathbb{C} e_{1}+\mathbb{C} u$ be the subspace generated by $e_{1}=(1,0, \ldots, 0)$ and $u$.

Corollary 3. We have $g_{n}(z, p, q)=g_{2}\left(\left(z_{1},\left\|z^{\prime}\right\|\right), p^{\star}, q^{\star}\right)$, where $p^{\star}=-q^{\star}=$ $(\beta, 0)$, and $g_{n}(\cdot, p, q) \in C^{1,1}\left(B^{n} \backslash\{p, q\}\right)$. Moreover, the function $g_{n}(\cdot, p, q)$ is real analytic in int $\Gamma_{p} \cup D \cup \operatorname{int} \Gamma_{q}$, and its first order partial derivatives extend continuously to $\partial B^{n}$. The leaves of the corresponding foliation are the following: The $z_{1}$-axis, complex lines through $p$ contained in $\Gamma_{p}$, complex lines through $q$ contained in $\Gamma_{q}$, and leaves $L_{u, \gamma} \subset V_{u} \cap B^{n}$ of the form

$$
L_{u, \gamma}: z=\lambda_{1} e_{1}+\lambda_{2} u, \gamma \lambda_{1}^{2}=\beta^{2}\left(\gamma-\lambda_{2}\right)\left(1-\bar{\gamma} \lambda_{2}\right),
$$

where $\gamma \in \Delta \backslash\{0\}, u=\left(0, u^{\prime}\right) \in \partial B^{n}$, which foliate $D \backslash z_{1}$-axis.

The proofs of Theorem 2 and Corollary 3 are given in Section 2 of the paper. We have seen above that $g_{n}(z, p, q)=g_{n}(z, p)$ for $z \in \Gamma_{p}$ and $g_{n}(z, p, q)=g_{n}(z, q)$ for $z \in \Gamma_{q}$. Similar results actually hold in the general case when the weights of the poles are arbitrary. We let $A=\{(p, \mu),(q, \nu)\} \subset$ $B^{n} \times(0,+\infty)$ and we denote by $g_{n}(\cdot, A)$ the pluricomplex Green function of $B^{n}$ with poles in $A$. Without loss of generality we can assume that $\mu \geq \nu$ and that $p=0$ and $q=(\alpha, 0, \ldots, 0), \alpha>0$, by using a suitable automorphism of $B^{n}$. For $u=\left(u_{1}, \ldots, u_{n}\right) \in \partial B^{n}$ we let $L_{u}=\{\zeta u: \zeta \in \Delta\}$ and $\Gamma_{0}=\bigcup\left\{L_{u}:\left|u_{1}\right| \leq \alpha / 2\right\}$. Moreover, we let $\Gamma_{q}=T\left(\Gamma_{0}\right)$, where $T \in \operatorname{Aut}\left(B^{n}\right)$ is an involution $(T \circ T=I d)$ satisfying $T(q)=0$ (see $[\mathbf{R}]$ ).

Proposition 4. In the above setting the following hold:

$$
\begin{aligned}
& g_{n}(z, A)=\mu g_{n}(z, 0) \quad \text { for } z \in \Gamma_{0}, \\
& \mu g_{n}(z, q) \leq g_{n}(z, A) \leq \nu g_{n}(z, q) \quad \text { for } z \in \Gamma_{q} .
\end{aligned}
$$

In particular, the function $z \rightarrow g_{n}(z, A)$ is not real analytic on $B^{n} \backslash\{0, q\}$ and hence is not of the form $g_{n}(z, A)=\log \|H(z)\|$, for any holomorphic map $H: B^{n} \rightarrow B^{n}$. Moreover, if $\mu>\nu$ then in general there is no complex line $L$ containing $q$ and such that $g_{n}(z, A)=\nu g_{n}(z, q)$ for all $z \in L \cap B^{n}$.

It was pointed out to us by L. Lempert that the existence of similar regions $\Gamma_{0}, \Gamma_{q}$ was noticed independently by $\mathrm{F}$. Wikström. As we shall see, the existence of $\Gamma_{0}$ follows from the following fact: If $L_{u} \subset \Gamma_{0}$ then there is a holomorphic map $F: B^{n} \rightarrow \Delta$ such that $F(q)=0$ and $F(\zeta u)=\zeta$ 
for all $\zeta \in \Delta$. Our next result can be viewed as a "partial converse" of this fact. Before we state it, we remark that such a map $F$ gives rise to a holomorphic retraction $r: B^{n} \rightarrow B^{n}$ onto $L_{u}, r(z)=F(z) u$, such that $r(p)=r(q)$. It is known that the holomorphic retracts of $B^{n}$ are precisely its affine subsets $[\mathbf{S}]$. However, there are examples in $[\mathbf{R}]$ showing that for a given holomorphic retract $X \subset B^{n}$ there are many retractions $r$ such that $r\left(B^{n}\right)=X$.

Theorem 5. Let $A=\{(0, \mu),(q, \nu)\} \subset B^{n} \times(0,+\infty)$, where $\mu \geq \nu$, and let $z_{0}=\zeta u$, for some $\zeta \in \Delta \backslash\{0\}$ and $u \in \partial B^{n}$. We have $g_{n}\left(z_{0}, A\right)=\mu g_{n}\left(z_{0}, 0\right)$ if and only if there exists a sequence of holomorphic functions $F_{j}: B^{n} \rightarrow \mathbb{C}$, $j=1,2, \ldots$, such that $F_{j}(q)=0$ for all $j \geq 1$ and the following hold:

(i) $F_{j}(t u)=t^{j}$, for all $t \in \Delta$ and for all $j \geq 1$.

(ii) $\lim \sup _{j \rightarrow \infty} \frac{1}{j} \log \left|F_{j}(z)\right| \leq g_{n}(z, A) / \mu$, for all $z \in B^{n}$.

The proofs of Proposition 4 and Theorem 5 are given in Section 3. We also make a remark there on what the analogue of Theorem 5 is in the case when $B^{n}$ is replaced by a smoothly bounded strongly convex domain (the proof remains the same as in the case of $B^{n}$ ).

Motivated by the above results we make the following:

Conjecture. If $\Omega$ is a bounded convex domain in $\mathbb{C}^{n}$ and $A$ is a finite subset of $\Omega \times(0,+\infty)$ then $g_{\Omega}(z, A)=\delta_{\Omega}^{A}(z)$ for all $z \in \Omega$.

Acknowledgement. The results of this paper constitute a part of my doctoral thesis. I am grateful to Professor John Erik Fornaess for all that he taught me in the field of several complex variables, and in particular for the stimulating discussions we had regarding this paper. I would also like to thank the referee for valuable suggestions and comments.

\section{Proof of Theorem 2.}

We begin by discussing the simplifications we use by arranging the poles $p$, $q$ in special positions. For $a \in B^{n}$ let us denote by $T_{a}$ the automorphism of $B^{n}$ defined as follows:

$$
T_{a}(z)=\frac{a-P_{a}(z)-\left(1-\|a\|^{2}\right)^{1 / 2} Q_{a}(z)}{1-\langle z, a\rangle},
$$

where $P_{a}(z)=\frac{\langle z, a\rangle}{\langle a, a\rangle} a$ is the projection onto $\mathbb{C} a$ and $Q_{a}=I d-P_{a}$ is the projection onto the orthogonal complement of $\mathbb{C} a$ (see $[\mathbf{R}]$ ). Since $g_{n}$ is biholomorphically invariant, by applying $T_{p}$ followed by a unitary transformation we can assume $p=0$ and $q=(\alpha, 0, \ldots, 0)$, for some $\alpha \in(0,1)$. If we set $a=\left(\alpha /\left(1+\sqrt{1-\alpha^{2}}\right), 0, \ldots, 0\right)$ then $T_{a}(p)=-T_{a}(q)=(\beta, 0, \ldots, 0)$, where $\beta=\alpha /\left(1+\sqrt{1-\alpha^{2}}\right) \in(0,1)$. So we may assume that $p=-q=(\beta, 0, \ldots, 0)$ for some $\beta \in(0,1)$. 
Let us now fix $n=2$ and assume that $p=-q=(\beta, 0)$. The proof of Theorem 2 goes as follows. We first compute $\delta_{2}\left(z_{0}, p, q\right)$ for all the points $z_{0}=(0, \gamma), \gamma \in \Delta$. We also show that for such points $z_{0}$ the extremal discs $f_{\gamma}: \Delta \rightarrow B^{2}$ for which the infimum in the definition (1.2) of $\delta_{2}\left(z_{0}, p, q\right)$ is attained are unique, properly embedded in $B^{2}$, and passing through both points $p, q$; moreover, we compute the discs $f_{\gamma}$ explicitly. Eliminating $\zeta$ from the equations $f_{\gamma}(\zeta)=z$ we obtain that

$$
f_{\gamma}(\Delta)=\left\{z \in B^{2}: \gamma z_{1}^{2}=\beta^{2}\left(\gamma-z_{2}\right)\left(1-\bar{\gamma} z_{2}\right)\right\} .
$$

We use this to show that the discs $f_{\gamma}$ foliate the domain $D$ defined by (1.6). Let us write $s_{\gamma}=f_{\gamma}^{-1}(p)>0$ and $t_{\gamma}=f_{\gamma}^{-1}(q)$; we will see that $t_{\gamma}=-s_{\gamma}$. If $z=f_{\gamma}\left(\zeta_{\star}\right)$ then using the $\operatorname{map} \zeta \rightarrow f_{\gamma}\left(\left(\zeta_{\star}-\zeta\right) /\left(1-\overline{\zeta_{\star}} \zeta\right)\right)$ it easily follows from the definition of $\delta_{2}(z, p, q)$ that

$$
\delta_{2}(z, p, q) \leq \log \left|\frac{s_{\gamma}^{2}-\zeta_{\star}^{2}}{1-s_{\gamma}^{2} \zeta_{\star}^{2}}\right|=g^{\star}(z) .
$$

As the curves $L_{\gamma}=f_{\gamma}(\Delta), \gamma \in \Delta$, foliate $D$, the function $g^{\star}$ introduced above is well defined on $D$. We extend $g^{\star}$ to $B^{2}$ by setting $g^{\star}(z)=g_{2}(z, p)$ for $z \in \Gamma_{p}$ and $g^{\star}(z)=g_{2}(z, q)$ for $z \in \Gamma_{q}$, where $\Gamma_{p}$ and $\Gamma_{q}$ are defined by (1.4) and (1.5). The function $g^{\star}$ is precisely the one given in the statement of Theorem 2. By the construction of $g^{\star}$ we clearly have

$$
g_{2}(z, p, q) \leq \delta_{2}(z, p, q)=\delta_{2}^{p, q}(z) \leq g^{\star}(z)
$$

for $z \in B^{2}$. Finally we show that $g^{\star}$ is a negative plurisubharmonic function on $B^{2}$ of class $C^{1,1}$, with logarithmic poles at $p$ and $q$ of weight one, hence $g^{\star} \leq g_{2}(\cdot, p, q)$. We also prove that the first order partial derivatives of $g_{2}(\cdot, p, q)$ are continuous up to the boundary of $B^{2}$.

We now proceed with the proof of Theorem 2. For $\gamma \in \Delta$ let us define $s_{\gamma} \in(0,1)$ by

$$
s_{\gamma}^{2}=\frac{\beta^{2}\left(1-|\gamma|^{2}\right)+\sqrt{\beta^{4}\left(1-|\gamma|^{2}\right)^{2}+4|\gamma|^{2}}}{2} .
$$

We also introduce the maps $f_{\gamma}: \Delta \rightarrow B^{2}$ given by

$$
f_{\gamma}(\zeta)=\left(\frac{s_{\gamma} \beta\left(1-|\gamma|^{2}\right) \zeta}{s_{\gamma}^{2}-|\gamma|^{2} \zeta^{2}}, \frac{\gamma\left(s_{\gamma}^{2}-\zeta^{2}\right)}{s_{\gamma}^{2}-|\gamma|^{2} \zeta^{2}}\right)
$$

Proposition 2.1. For $z_{0}=(0, \gamma) \in B^{2}$ we have $\delta_{2}\left(z_{0}, p, q\right)=\log s_{\gamma}^{2}$. Moreover, the functions $f_{\gamma}$ are the unique extremal discs realizing the infimum in the definition (1.2) of $\delta_{2}\left(z_{0}, p, q\right)$. The map $f_{\gamma}: \Delta \rightarrow B^{2}$ is proper, injective, non-singular in $\Delta$, and $f_{\gamma}(0)=z_{0}, f_{\gamma}\left(s_{\gamma}\right)=p, f_{\gamma}\left(-s_{\gamma}\right)=q$.

Proof. We deal first with the case $\gamma=0$. By considering the maps $\zeta \rightarrow(\zeta, 0)$ and $z=\left(z_{1}, z_{2}\right) \rightarrow z_{1}$ we get $g_{2}(0, p, q)=\delta_{2}(0, p, q)=\log \beta^{2}$. If $f=\left(f_{1}, f_{2}\right)$ : 
$\Delta \rightarrow B^{2}$ satisfies $f(0)=0, f(s)=p, f(t)=q$ and $s|t|=\beta^{2}$, then $f_{1}(\zeta)=\zeta$ by the Schwarz lemma, so $f_{2}=0$.

For the remainder of the proof we assume $\gamma \neq 0$. Motivated by the definition of $\delta_{2}\left(z_{0}, p, q\right)$ we define $S$ to be the set of all pairs $(s, t) \in(0,1) \times \Delta$ for which there exists a holomorphic map $f: \Delta \rightarrow B^{2}$ satisfying $f(0)=z_{0}$, $f(s)=p, f(t)=q$. We divide the proof in two steps. In the first one we show that the function $(s, t) \in S \rightarrow s|t|$ has a unique minimum point at $\left(s_{\gamma},-s_{\gamma}\right) \in S$, where $s_{\gamma}$ is defined by $(2.2)$, and that $s_{\gamma}^{2}=\inf \{s|t|:(s, t) \in$ $S\}$; so $\delta_{2}\left(z_{0}, p, q\right)=\log s_{\gamma}^{2}$. In the second step we prove that there exists a unique disc $f_{\gamma}: \Delta \rightarrow B^{2}$ corresponding to the point $\left(s_{\gamma},-s_{\gamma}\right)$, that $f_{\gamma}$ is proper and it is given by (2.3). The facts that $f_{\gamma}$ is injective and that $f_{\gamma}^{\prime}(\zeta) \neq 0$ on $\Delta$ now follow by inspection.

Step 1. Let us introduce the following notations:

$$
c=\left(1-|\gamma|^{2}\right) \beta^{2}+|\gamma|^{2}, \quad d=\left(1-|\gamma|^{2}\right) \beta^{2}-|\gamma|^{2} .
$$

We also define for $s \in(0,1)$ and $t \in \Delta$

$$
E(s, t)=\frac{\left(s^{2}-c\right)\left(|t|^{2}-c\right)}{|s t+d|^{2}}-\frac{\left(1-s^{2}\right)\left(1-|t|^{2}\right)}{|1-s t|^{2}} .
$$

We first prove that

$$
S=\left\{(s, t) \in(0,1) \times \Delta: s \neq t, s^{2}>c,|t|^{2}>c, E(s, t) \geq 0\right\} .
$$

Let $(s, t) \in S$ and let $f: \Delta \rightarrow B^{2}$ be such that $f(0)=z_{0}, f(s)=p, f(t)=q$. Clearly $s \neq t$. Let $T$ be given by (2.1) such that $T\left(z_{0}\right)=0$ :

$$
T\left(z_{1}, z_{2}\right)=\left(\left(1-|\gamma|^{2}\right)^{1 / 2} \frac{-z_{1}}{1-\bar{\gamma} z_{2}}, \frac{\gamma-z_{2}}{1-\bar{\gamma} z_{2}}\right) .
$$

Then the analytic disc $T f: \Delta \rightarrow B^{2}$ satisfies $T f(0)=0$ and

$$
T f(s)=\widetilde{p}=\left(-\left(1-|\gamma|^{2}\right)^{1 / 2} \beta, \gamma\right), \quad T f(t)=\widetilde{q}=\left(\left(1-|\gamma|^{2}\right)^{1 / 2} \beta, \gamma\right) .
$$

Hence $T f(\zeta)=\zeta \widetilde{f}(\zeta)$, where $\widetilde{f}: \Delta \rightarrow \overline{B^{2}}$. We have $\widetilde{f}(s)=\widetilde{p} / s, \widetilde{f}(t)=\widetilde{q} / t$, so $c=\|\widetilde{p}\|^{2} \leq s^{2}$ and $c=\|\widetilde{q}\|^{2} \leq|t|^{2}$. If $s=\sqrt{c}=\|\widetilde{p}\|$ it follows by Lemma 2.2 that $T f(\zeta)=\zeta \widetilde{p} /\|\widetilde{p}\|$ for all $\zeta \in \Delta$. As $\gamma \neq 0$ this implies $\widetilde{q} \notin T f(\Delta)$, a contradiction. Hence we have $s^{2}>c$ and similarly $|t|^{2}>c$, so $\widetilde{f}(\Delta) \subset B^{2}$. Let $\Phi$ be the automorphism of $B^{2}$ of form (2.1) determined by $\Phi(\widetilde{p} / s)=0$. By the Schwarz lemma applied to $\Phi \circ \widetilde{f}$ we get

$$
\left\|\Phi\left(\frac{\widetilde{q}}{t}\right)\right\| \leq\left|\frac{t-s}{1-s t}\right|
$$

which is equivalent to $E(s, t) \geq 0$.

Thus we have shown that $(s, t) \in S$ implies $s \neq t, s^{2}>c,|t|^{2}>c$, and $E(s, t) \geq 0$. Conversely, we assume that $s, t$, with $s \neq t$, satisfy these three 
inequalities and we let $\widetilde{p}, \widetilde{q}, T$ and $\Phi$ be as defined above. Then the analytic $\operatorname{disc} f: \Delta \rightarrow B^{2}$,

$$
f(\zeta)=T\left(\zeta \Phi\left(\frac{1-s t}{t-s} \frac{\zeta-s}{1-s \zeta} \Phi\left(\frac{\widetilde{q}}{t}\right)\right)\right),
$$

is well defined and satisfies $f(0)=T(0)=z_{0}, f(s)=T(s \Phi(0))=T(\widetilde{p})=p$, and $f(t)=T(\widetilde{q})=q$. So relation (2.4) is completely proved.

Let $\bar{S} \subset(0,1] \times \bar{\Delta}$ denote the closure of $S$. Since $\bar{S}$ is compact we can find a point $\left(s_{0}, t_{0}\right) \in \bar{S}$ such that $s_{0}\left|t_{0}\right|=\min \{s|t|: \quad(s, t) \in \bar{S}\}$. We show that there is a unique point $\left(s_{0}, t_{0}\right)$ with this property and that $\left(s_{0}, t_{0}\right)=\left(s_{\gamma},-s_{\gamma}\right) \in S$. If $s_{0}^{2}=c$ then $E\left(s_{0}, t_{0}\right) \geq 0$ implies $\left|t_{0}\right|=1$, so $s_{0}\left|t_{0}\right|=\sqrt{c}$; a similar conclusion holds if $\left|t_{0}\right|^{2}=c$. We also note that $s_{0} \neq t_{0}$; indeed, we have $E(s, s)<0$ for all $s>0$ with $s^{2} \in[c, 1]$. From this discussion it follows that

$$
s_{0}\left|t_{0}\right|=\sqrt{c} \text { if }\left(s_{0}, t_{0}\right) \in \bar{S} \backslash S .
$$

We assume now that $\left(s_{0}, t_{0}\right) \in S$. Then $\left(s_{0}, t_{0}\right)$ is a solution of the problem:

$$
\left\{\begin{array}{l}
s|t| \rightarrow \min , \\
(s, t) \in(0,1) \times \Delta, s \neq t, s^{2}>c,|t|^{2}>c, E(s, t) \geq 0 .
\end{array}\right.
$$

Clearly we must have $E\left(s_{0}, t_{0}\right)=0$. Moreover, if $t$ lies on the circle $|t|=\left|t_{0}\right|$ we must have $E\left(s_{0}, t\right) \leq 0$ (otherwise one could find $t^{\prime} \neq s_{0}$ such that $\left|t^{\prime}\right|^{2}>c,\left|t^{\prime}\right|<\left|t_{0}\right|$ and $E\left(s_{0}, t^{\prime}\right)>0$, hence $s_{0}\left|t^{\prime}\right|<s_{0}\left|t_{0}\right|$, a contradiction). By the definition of $E(s, t)$ we have that $E\left(s_{0}, t\right) \leq 0$ is equivalent to

$$
\left|\frac{s_{0} t+d}{1-s_{0} t}\right|^{2} \geq \frac{\left(s_{0}^{2}-c\right)\left(\left|t_{0}\right|^{2}-c\right)}{\left(1-s_{0}^{2}\right)\left(1-\left|t_{0}\right|^{2}\right)},
$$

and equality holds for $t=t_{0}$. The image of the circle $|t|=\left|t_{0}\right|$ under the map

$$
t \rightarrow \frac{s_{0} t+d}{1-s_{0} t}
$$

is a circle $C$ orthogonal to the real axis and centered at

$$
X_{C}=\frac{s_{0}^{2}\left|t_{0}\right|^{2}+d}{1-s_{0}^{2}\left|t_{0}\right|^{2}} .
$$

We have two cases:

Case 1. $X_{C} \neq 0$. We have by the formula above that $\left(s_{0} t_{0}+d\right) /\left(1-s_{0} t_{0}\right)$ is the point on the circle $C$ of smallest magnitude. It follows that $\left(s_{0} t_{0}+\right.$ $d) /\left(1-s_{0} t_{0}\right)$, and hence $t_{0}$, are real. For $t$ real we note that $E(s, t)=0$ is equivalent to $F(s, t)=0$, where

$$
F(s, t)=\left(s^{2}-c\right)\left(t^{2}-c\right)(1-s t)^{2}-\left(1-s^{2}\right)\left(1-t^{2}\right)(s t+d)^{2} .
$$


By $(2.8)$ we see that $\left(s_{0}, t_{0}\right)$ is a solution of the problem

$$
\left\{\begin{array}{l}
s^{2} t^{2} \rightarrow \min \\
(s, t) \in(0,1) \times(-1,1), s \neq t, s^{2}>c, t^{2}>c, F(s, t)=0 .
\end{array}\right.
$$

We make the change of coordinates $x=s+t, y=s t$. This is a local diffeomorphism away from the diagonal $s=t$. In the new coordinates the image of the curve $F(s, t)=0$ is the curve $\widetilde{F}(x, y)=0$, where

$$
\widetilde{F}(x, y)=(y+c)^{2}(1-y)^{2}-(y+1)^{2}(y+d)^{2}+\left[(y+d)^{2}-c(1-y)^{2}\right] x^{2} .
$$

We let $x_{0}=s_{0}+t_{0}, y_{0}=s_{0} t_{0}$. Then $\left(x_{0}, y_{0}\right)$ is a local solution of the problem

$$
\left\{\begin{array}{l}
y^{2} \rightarrow \min \\
\widetilde{F}(x, y)=0 .
\end{array}\right.
$$

Hence we must have

$$
\frac{\partial \widetilde{F}}{\partial x}\left(x_{0}, y_{0}\right)=0 \Rightarrow\left(y_{0}+d\right)^{2}=c\left(1-y_{0}\right)^{2} \text { or } x_{0}=0 .
$$

If $\left(y_{0}+d\right)^{2}=c\left(1-y_{0}\right)^{2}$ then using $\widetilde{F}\left(x_{0}, y_{0}\right)=0$ we get $\left(y_{0}+c\right)^{2}=$ $c\left(y_{0}+1\right)^{2}$, hence $y_{0}^{2}=c$. But the system of equations $\left(y_{0}+d\right)^{2}=c\left(1-y_{0}\right)^{2}$, $y_{0}^{2}=c$ has no solution.

If $x_{0}=0$ then $s_{0}=-t_{0}$ and hence $s_{0}^{2}=-y_{0}$. The equation $\widetilde{F}\left(x_{0}, y_{0}\right)=0$ implies that $\left(y_{0}+c\right)\left(1-y_{0}\right)=-\left(y_{0}+1\right)\left(y_{0}+d\right)$ or $\left(y_{0}+c\right)\left(1-y_{0}\right)=$ $\left(y_{0}+1\right)\left(y_{0}+d\right)$. If the former equality holds we get $y_{0}=-\beta^{2}$, which is in contradiction to $s_{0}^{2}=-y_{0}>c$. If the latter equality holds we solve for $y_{0}$ and obtain $y_{0}=-s_{\gamma}^{2}$, where $s_{\gamma}$ is defined by (2.2). As $s_{0}^{2}=s_{\gamma}^{2}>c$ we see that $\left(s_{\gamma},-s_{\gamma}\right) \in S$ is the only possible solution of problem (2.8) in Case 1.

Case 2. $X_{C}=0$. Then $s_{0}^{2}\left|t_{0}\right|^{2}=-d>0$ and the equation $E\left(s_{0}, t_{0}\right)=0$ becomes

$$
\left|\frac{s_{0} t_{0}+d}{1-s_{0} t_{0}}\right|^{2}=\frac{-d-c\left(s_{0}^{2}+\left|t_{0}\right|^{2}\right)+c^{2}}{-d-\left(s_{0}^{2}+\left|t_{0}\right|^{2}\right)+1} .
$$

Writing $s_{0} t_{0}=\sqrt{-d} e^{i \theta}$ we get that the left hand side of the above equality is equal to $-d$. Using this and substituting $x_{0}=s_{0}^{2}+\left|t_{0}\right|^{2}$ in the equation $E\left(s_{0}, t_{0}\right)=0$ we get $x_{0}=c-d$. Since $s_{0}^{2}>c$ and $\left|t_{0}\right|^{2}>c$ we have $c-d=x_{0}>2 c$, a contradiction.

We conclude by above that the problem (2.8) has at most one solution; hence if $\left(s_{0}, t_{0}\right) \in S$ we have shown that $\left(s_{0}, t_{0}\right)=\left(s_{\gamma},-s_{\gamma}\right)$. One can easily check that $s_{\gamma}^{2}<\sqrt{c}$, so in view of $(2.7)$ the function $(s, t) \in S \rightarrow s|t|$ has a unique minimum at $\left(s_{\gamma},-s_{\gamma}\right) \in S$ and $s_{\gamma}^{2}=\inf \{s|t|:(s, t) \in S\}$.

Step 2. Let $f: \Delta \rightarrow B^{2}$ be a holomorphic map verifying $f(0)=z_{0}$, $f\left(s_{\gamma}\right)=p, f\left(-s_{\gamma}\right)=q$. By Theorem 4 and Lemma 7 of [AT1] $f$ is unique with these properties and proper: Indeed, with notations from [AT1] $f$ is extremal for $\rho_{1}(s), s=\left\{z_{0}, p, q\right\}$, hence norm minimal among holomorphic 
maps $\Delta \rightarrow \mathbb{C}^{n}$ with $\left\{0, s_{\gamma},-s_{\gamma}\right\} \rightarrow\left\{z_{0}, p, q\right\}$. We call this map $f_{\gamma}$ and find it explicitly as follows. Let for $\zeta \in \Delta$

$$
\tilde{f}_{\gamma}(\zeta)=\left(-\frac{\beta\left(1-|\gamma|^{2}\right)^{1 / 2}}{s_{\gamma}}, \frac{\gamma}{s_{\gamma}^{2}} \zeta\right) .
$$

Then $\widetilde{f}_{\gamma}\left(s_{\gamma}\right)=\widetilde{p} / s_{\gamma}, \widetilde{f}_{\gamma}\left(-s_{\gamma}\right)=-\widetilde{q} / s_{\gamma}$. By the definition $(2.2)$ of $s_{\gamma}^{2}$ we see that

$$
s_{\gamma}^{4}-\beta^{2}\left(1-|\gamma|^{2}\right) s_{\gamma}^{2}-|\gamma|^{2}=0,
$$

which shows $\tilde{f}_{\gamma}(\Delta) \subset B^{2}$. Next we let $f_{\gamma}(\zeta)=T\left(\zeta \widetilde{f}_{\gamma}(\zeta)\right)$, with $T$ given by (2.5). A simple computation shows that the map $f_{\gamma}$ has the form (2.3) and it satisfies $f_{\gamma}(0)=z_{0}, f_{\gamma}\left(s_{\gamma}\right)=p, f_{\gamma}\left(-s_{\gamma}\right)=q$. This concludes the proof of Proposition 2.1.

We have used the following well known variant of the Schwarz lemma:

Lemma 2.2. Assume that the holomorphic map $f: \Delta \rightarrow B^{2}$ satisfies $f(0)=0$ and $f(\|z\|)=z$ for some $z \in B^{2} \backslash\{0\}$. Then $f(\zeta)=\zeta z /\|z\|$, for all $\zeta \in \Delta$.

For $z \in D$ we define

$$
\begin{aligned}
& x(z)=\frac{z_{2}}{\left|z_{2}\right|^{4}-\left|1-z_{1}^{2} / \beta^{2}\right|^{2}}\left(\left|z_{2}\right|^{2}-1+\frac{\bar{z}_{1}^{2}}{\beta^{2}}\right), \\
& \gamma(z)=\frac{1-\sqrt{1-4|x(z)|^{2}}}{2|x(z)|^{2}} x(z) .
\end{aligned}
$$

Proposition 2.3. The complex curves $L_{\gamma}=f_{\gamma}(\Delta)$, where $f_{\gamma}$ is defined by (2.3) and $\gamma \in \Delta$, are given by

$$
L_{\gamma}=\left\{z \in B^{2}: \gamma z_{1}^{2}=\beta^{2}\left(\gamma-z_{2}\right)\left(1-\bar{\gamma} z_{2}\right)\right\} .
$$

We have $L_{\gamma} \subset D \cup\{p, q\}$ for all $\gamma \in \Delta$. Moreover, for $z \in D$ the number $\gamma(z)$ defined by (2.11) is the unique solution in $\Delta$ of the equation in (2.12), hence the curves $L_{\gamma}, \gamma \in \Delta$, foliate $D$.

Proof. Using the expression of the second coordinate of $f_{\gamma}(\zeta)=\left(z_{1}, z_{2}\right)$ we write $\zeta^{2}$ in terms of $z_{2}$ and obtain

$$
\zeta^{2}=\frac{s_{\gamma}^{2}}{\gamma} \frac{\gamma-z_{2}}{1-\bar{\gamma} z_{2}}
$$

Formula (2.12) now follows by squaring the formula of $z_{1}$ from $f_{\gamma}(\zeta)=$ $\left(z_{1}, z_{2}\right)$ and by using the above formula for $\zeta^{2}$.

For the rest of the proof it is convenient to introduce

$$
x=\frac{\gamma}{1+|\gamma|^{2}} \text {. }
$$


Using this substitution, the equation in (2.12) can be written in the form

$$
x\left(1-\frac{z_{1}^{2}}{\beta^{2}}\right)+\bar{x} z_{2}^{2}=z_{2} .
$$

Let us assume that for some $\gamma \in \Delta$ we have $L_{\gamma} \backslash D \neq\{p, q\}$, or, without loss of generality, that $L_{\gamma} \cap \Gamma_{p} \neq\{p\}$. Then there is $z \in L_{\gamma} \backslash\{p\}$ such that $\beta z_{2}=e^{i \theta}\left(\beta-z_{1}\right)$; in particular, this implies that $\Re z_{1}>0$. Writing $\mu=z_{1} / \beta$, $z_{2}=e^{i \theta}(1-\mu)$, and $c=x e^{-i \theta}$, Equation $(2.15)$ becomes $c(1+\mu)+\bar{c}(1-\mu)=$ 1. As $\Re \mu>0$ it follows that $\Im c=0$, hence $c=1 / 2$; this is in contradiction with $|c|=|x|<1 / 2$, which holds since $\gamma \in \Delta$ (see (2.14)). We conclude that $L_{\gamma} \subset D \cup\{p, q\}$ for all $\gamma \in \Delta$.

For a fixed $z \in D$ we now consider the Equation (2.15). The determinant of the corresponding system of two real equations is $\left|1-z_{1}^{2} / \beta^{2}\right|^{2}-\left|z_{2}\right|^{4}>0$, since $z \in D$; hence Equation (2.15) has a unique solution $x=x(z)$ for $z \in D$, where $x(z)$ is given by (2.10). Let us write again $\mu=z_{1} / \beta \in \mathbb{C}$, $r=\left|z_{2}\right| \in[0,1)$. Since $z \in D$ we have $r<\min \{|1-\mu|,|1+\mu|\}$. We claim that this implies

$$
|x(z)|=\frac{r\left|r^{2}-1+\mu^{2}\right|}{\left|1-\mu^{2}\right|^{2}-r^{4}}<\frac{1}{2} .
$$

The proof of this claim is given in Lemma 2.4. For $x=x(z)$ with $|x|<1 / 2$ we notice that Equation (2.14) has a unique solution $\gamma=\gamma(z) \in \Delta$ : Indeed, $\arg \gamma=\arg x$, and $|\gamma|$ is the unique solution of $|x||\gamma|^{2}-|\gamma|+|x|=0$ contained in $[0,1)$. We conclude that for $z \in D$ the equation in (2.12) has a unique solution $\gamma(z) \in \Delta$, given by (2.11).

Lemma 2.4. Let $r \in[0,1)$ and $\mu \in \mathbb{C}$ be such that $r<\min \{|1-\mu|,|1+\mu|\}$. Then

$$
\frac{r\left|r^{2}-1+\mu^{2}\right|}{\left|1-\mu^{2}\right|^{2}-r^{4}}<\frac{1}{2}
$$

Proof. An elementary proof of this inequality is given in $[\mathbf{C}]$. We are grateful to the referee, who suggested the following argument. Consider the following:

$$
\begin{aligned}
& F_{1}=\left\{\left(\gamma, s_{\gamma}\right): \gamma \in \Delta\right\}, F_{2}=\left\{\left(\gamma,-s_{\gamma}\right): \gamma \in \Delta\right\} \\
& G=\left\{(\gamma, \zeta) \in \Delta^{2}: \zeta \neq \pm s_{\gamma}\right\}=\Delta^{2} \backslash\left(F_{1} \cup F_{2}\right), \\
& \Phi: G \rightarrow D, \Phi(\gamma, \zeta)=f_{\gamma}(\zeta)
\end{aligned}
$$

Then $G$ is open, and, by arguments in the proof of Proposition 2.3, the map $\Phi$ is well-defined, continuous and one-to-one. Hence $\Phi$ is a homeomorphism onto $\Phi(G)$. We will check that $\Phi$ is proper, which implies $\Phi(G)=D$. This is equivalent to the inequality we seek, $|x(z)|<1 / 2$ for $z \in D$, as (2.14) has solutions if and only if $|x|<1 / 2$. To show $\Phi$ is proper we assume that $(\gamma, \zeta) \in G \rightarrow\left(\gamma_{0}, \zeta_{0}\right) \in \partial G=F_{1} \cup F_{2} \cup(\partial \Delta \times \Delta) \cup(\Delta \times \partial \Delta) \cup(\partial \Delta \times \partial \Delta)$. 
If $\left(\gamma_{0}, \zeta_{0}\right) \in F_{1} \cup F_{2}$ then $\Phi(\gamma, \zeta) \rightarrow(\beta, 0) \in \partial D$, or $\Phi(\gamma, \zeta) \rightarrow(-\beta, 0) \in$ $\partial D$.

If $\left(\gamma_{0}, \zeta_{0}\right)=\left(e^{i \theta}, \zeta_{0}\right) \in \partial \Delta \times \Delta$ then $\Phi(\gamma, \zeta) \rightarrow \partial D$ by $(2.3)$.

If $\left(\gamma_{0}, \zeta_{0}\right)=\left(\gamma_{0}, e^{i \phi}\right) \in \Delta \times \partial \Delta$ then $\Phi(\gamma, \zeta) \rightarrow \partial D$, as $f_{\gamma_{0}}$ is proper.

If $\left(\gamma_{0}, \zeta_{0}\right)=\left(e^{i \theta}, e^{i \phi}\right)$ and $\phi \neq 0, \pi$, then $s_{\gamma}^{2}-|\gamma|^{2} \zeta^{2} \rightarrow 1-e^{2 i \phi} \neq 0$, so $\Phi(\gamma, \zeta) \rightarrow\left(0, e^{i \theta}\right) \in \partial D$. If $\phi=0$ then let $\left(z_{1}, z_{2}\right)=f_{\gamma}(\zeta)$. A simple computation yields

$$
\frac{\beta-z_{1}}{\beta z_{2}}=\frac{s_{\gamma}+\zeta-\zeta\left(1-|\gamma|^{2}\right)}{\gamma\left(s_{\gamma}+\zeta\right)} \rightarrow e^{-i \theta}, \text { as }(\gamma, \zeta) \rightarrow\left(e^{i \theta}, 1\right) .
$$

Hence $\Phi(\gamma, \zeta) \rightarrow \partial D$. A similar argument works in the case $\phi=\pi$.

Since the maps $f_{\gamma}$ defined by (2.3) are injective and their images $f_{\gamma}(\Delta)$, $\gamma \in \Delta$, foliate $D$, we can define a function $g^{\star}$ on $D$ as follows: For each $z \in D$ there is a unique $\gamma=\gamma(z) \in \Delta$ (given by (2.11)) such that $z \in f_{\gamma}(\Delta)$, and hence a unique $\zeta=\zeta(z) \in \Delta$ (given by (2.13)) such that $z=f_{\gamma}(\zeta)$. So we can define

$$
g^{\star}(z)=\log \left|\frac{s_{\gamma}^{2}-\zeta^{2}}{1-s_{\gamma}^{2} \zeta^{2}}\right| .
$$

The function $\zeta \rightarrow g^{\star}\left(f_{\gamma}(\zeta)\right)$ is clearly harmonic in $\Delta \backslash\left\{-s_{\gamma}, s_{\gamma}\right\}$, for all $\gamma \in$ $\Delta$. As we noticed at the beginning of this section, we have $\delta_{2}(z, p, q) \leq g^{\star}(z)$ for $z \in D$. Using (2.13) and the above formula of $g^{\star}$ we obtain

$$
g^{\star}(z)=\log \frac{\left|z_{2}\right|}{\left|\gamma / s_{\gamma}^{2}-\beta^{2} \gamma+\beta^{2} z_{2}\right|},
$$

where $z \in D, \gamma=\gamma(z)$ is given by (2.11), and $s_{\gamma}^{2}$ is given by (2.2) (we point out that in the above computation we use relation (2.9) to replace $s_{\gamma}^{4}$ in terms of $s_{\gamma}^{2}$, which allows us to simplify with a factor of $\left.\left(1-|\gamma|^{2}\right)\right)$.

Lemma 2.5. For $z \in D$ we have

$$
g^{\star}(z)=\frac{1}{2} \log \frac{\left|\beta^{2}-z_{1}^{2}\right|^{2}+\beta^{4}\left|z_{2}\right|^{4}+2\left(1-\beta^{4}\right)\left|z_{2}\right|^{2}+\sqrt{M(z)}}{2\left|1-\beta^{2} z_{1}^{2}\right|^{2}},
$$

where $M(z)=\left(\beta^{4}\left|z_{2}\right|^{4}-\left|\beta^{2}-z_{1}^{2}\right|^{2}\right)^{2}+\left.4\left(1-\beta^{4}\right)\left|z_{2}\right|^{2}\left|\beta^{2}\right| z_{2}\right|^{2}-\left.\left(\beta^{2}-z_{1}^{2}\right)\right|^{2}$.

Proof. We first use relation (2.11) to replace $\gamma=\gamma(x)$ in (2.16), and then we write down a formula of $g^{\star}$ in terms of $z$ alone by using (2.10). We now present the main steps of the computation. Using (2.11) and (2.14) we have

$$
\frac{\gamma}{1-|\gamma|^{2}}=\frac{1+|\gamma|^{2}}{1-|\gamma|^{2}} x=\frac{x}{\sqrt{1-4|x|^{2}}} .
$$


To compute $\gamma / s_{\gamma}^{2}$ we write it in terms of $\gamma /\left(1-|\gamma|^{2}\right)$; using the above relation and (2.11) again we get

$$
\frac{\gamma}{s_{\gamma}^{2}}-\beta^{2} \gamma=\frac{\sqrt{\beta^{4}+4\left(1-\beta^{4}\right)|x|^{2}}-\beta^{2}}{2 \bar{x}} .
$$

Replacing this in the formula (2.16) of $g^{\star}$ we see that

$$
g^{\star}(z)=\log \frac{2\left|z_{2}\right||x|}{\left|\sqrt{\beta^{4}+4\left(1-\beta^{4}\right)|x|^{2}}-\beta^{2}+2 \beta^{2} z_{2} \bar{x}\right|} .
$$

Using formula (2.10) we get

$$
\beta^{4}+4\left(1-\beta^{4}\right)|x|^{2}=\frac{\beta^{4}}{\left(\left|\beta^{2}-z_{1}^{2}\right|^{2}-\beta^{4}\left|z_{2}\right|^{4}\right)^{2}} M(z),
$$

and hence

$$
g^{\star}(z)=\log \frac{\left.2\left|z_{2}\right|^{2}\left|\beta^{2}\right| z_{2}\right|^{2}-\left(\beta^{2}-z_{1}^{2}\right) \mid}{\left|\sqrt{M(z)}-\left[\beta^{4}\left|z_{2}\right|^{4}-2 \beta^{2}\left|z_{2}\right|^{2}\left(\beta^{2}-z_{1}^{2}\right)+\left|\beta^{2}-z_{1}^{2}\right|^{2}\right]\right|} .
$$

A simple computation now yields

$$
\begin{aligned}
M & (z)-\left[\beta^{4}\left|z_{2}\right|^{4}-2 \beta^{2}\left|z_{2}\right|^{2}\left(\beta^{2}-z_{1}^{2}\right)+\left|\beta^{2}-z_{1}^{2}\right|^{2}\right]^{2} \\
= & 4 \beta^{2}\left|z_{2}\right|^{2}\left(\left|\beta^{2}-z_{1}^{2}\right|^{2}-\beta^{2}\left|z_{2}\right|^{2}\left(\beta^{2}-z_{1}^{2}\right)\right)\left(\beta^{2}-z_{1}^{2}-\beta^{2}\left|z_{2}\right|^{2}\right) \\
& +\left.\left.4\left(1-\beta^{4}\right)\left|z_{2}\right|^{2}\left|\beta^{2}-z_{1}^{2}-\beta^{2}\right| z_{2}\right|^{2}\right|^{2} \\
= & \left.\left.4\left|z_{2}\right|^{2}\left(1-\beta^{2} z_{1}^{2}\right)\left|\beta^{2}-z_{1}^{2}-\beta^{2}\right| z_{2}\right|^{2}\right|^{2} .
\end{aligned}
$$

Using this and the last formula of $g^{\star}$ we get

$$
g^{\star}(z)=\log \frac{\left.\left|\sqrt{M(z)}+\beta^{4}\right| z_{2}\right|^{4}-2 \beta^{2}\left|z_{2}\right|^{2}\left(\beta^{2}-z_{1}^{2}\right)+\left|\beta^{2}-z_{1}^{2}\right|^{2} \mid}{\left.2\left|1-\beta^{2} z_{1}^{2}\right|\left|\beta^{2}-z_{1}^{2}-\beta^{2}\right| z_{2}\right|^{2} \mid}=\log \frac{N_{1}}{N_{2}} \text {. }
$$

The final step is to compute $N_{1}^{2}$ :

$$
\begin{aligned}
N_{1}^{2}= & |\sqrt{M(z)}+| \beta^{2}\left|z_{2}\right|^{2}-\left.\left(\beta^{2}-z_{1}^{2}\right)\right|^{2}-\left.2 \beta^{2}\left|z_{2}\right|^{2} i \Im\left(\beta^{2}-z_{1}^{2}\right)\right|^{2} \\
= & M(z)+\left.\left|\beta^{2}\right| z_{2}\right|^{2}-\left.\left(\beta^{2}-z_{1}^{2}\right)\right|^{4}+4 \beta^{4}\left|z_{2}\right|^{4}\left[\Im\left(\beta^{2}-z_{1}^{2}\right)\right]^{2} \\
& +\left.2\left|\beta^{2}\right| z_{2}\right|^{2}-\left.\left(\beta^{2}-z_{1}^{2}\right)\right|^{2} \sqrt{M(z)} .
\end{aligned}
$$

Using

$$
\begin{aligned}
& \left(\beta^{4}\left|z_{2}\right|^{4}-\left|\beta^{2}-z_{1}^{2}\right|^{2}\right)^{2}+4 \beta^{4}\left|z_{2}\right|^{4}\left[\Im\left(\beta^{2}-z_{1}^{2}\right)\right]^{2} \\
& =\left(\beta^{4}\left|z_{2}\right|^{4}+\left|\beta^{2}-z_{1}^{2}\right|^{2}\right)^{2}-4 \beta^{4}\left|z_{2}\right|^{4}\left[\Re\left(\beta^{2}-z_{1}^{2}\right)\right]^{2} \\
& =\left.\left|\beta^{2}\right| z_{2}\right|^{2}-\left.\left.\left(\beta^{2}-z_{1}^{2}\right)\right|^{2}\left|\beta^{2}\right| z_{2}\right|^{2}+\left.\left(\beta^{2}-z_{1}^{2}\right)\right|^{2}
\end{aligned}
$$


we get

$$
\begin{aligned}
N_{1}^{2}=\left.2\left|\beta^{2}\right| z_{2}\right|^{2}-\left.\left(\beta^{2}-z_{1}^{2}\right)\right|^{2} & \\
& \cdot\left[\beta^{4}\left|z_{2}\right|^{4}+\left|\beta^{2}-z_{1}^{2}\right|^{2}+2\left(1-\beta^{4}\right)\left|z_{2}\right|^{2}+\sqrt{M(z)}\right] .
\end{aligned}
$$

We conclude that $g^{\star}(z)=\frac{1}{2} \log \frac{N_{1}^{2}}{N_{2}^{2}}$ has the desired form.

We now extend the function $g^{\star}$ to $B^{2}$ by defining

$$
\begin{aligned}
& g^{\star}(z)=g_{2}(z, p)=\log \frac{\sqrt{\left|\beta-z_{1}\right|^{2}+\left(1-\beta^{2}\right)\left|z_{2}\right|^{2}}}{\left|1-\beta z_{1}\right|}, \text { for } z \in \Gamma_{p}, \\
& g^{\star}(z)=g_{2}(z, q)=\log \frac{\sqrt{\left|\beta+z_{1}\right|^{2}+\left(1-\beta^{2}\right)\left|z_{2}\right|^{2}}}{\left|1+\beta z_{1}\right|}, \text { for } z \in \Gamma_{q},
\end{aligned}
$$

where $\Gamma_{p}, \Gamma_{q}$ are defined by (1.4) and (1.5) respectively (the formula of $g_{2}(z, p)$ is well known, and it can be easily obtained from the results we recalled in the Introduction and from formula (2.1)). The final part in the proof of Theorem 2 is to show that the function $g^{\star}$ is plurisubharmonic in $B^{2}$ and of class $C^{1,1}$ on $B^{2} \backslash\{p, q\}$. The function $g^{\star}$ is clearly plurisubharmonic in int $\Gamma_{p} \cup \operatorname{int} \Gamma_{q}$. We next prove that $g^{\star}$ is plurisubharmonic in $D$. To this end we consider the holomorphic mapping $F: D \rightarrow \mathbb{C} \times \Delta, F(z)=(u, w)$, where

$$
u=\sqrt{2\left(1-\beta^{4}\right)} \frac{z_{2}}{\beta^{2}-z_{1}^{2}}, \quad w=\frac{\beta^{2} z_{2}^{2}}{\beta^{2}-z_{1}^{2}} .
$$

We also define a function $V$ on $\mathbb{C} \times \Delta$ by

$$
\begin{aligned}
& V(u, w) \\
& =\log \left(\sqrt{1+|u|^{2}+|w|^{2}+\left|u^{2}+2 w\right|}+\sqrt{1+|u|^{2}+|w|^{2}-\left|u^{2}+2 w\right|}\right) .
\end{aligned}
$$

Lemma 2.6. For $z \in D$ we have

$$
g^{\star}(z)=\log \left|\frac{\beta^{2}-z_{1}^{2}}{1-\beta^{2} z_{1}^{2}}\right|+V(F(z))-\log 2 .
$$

Proof. Let $M(z)$ be as in Lemma 2.5. Then

$$
\begin{aligned}
M(z) & =\left|\beta^{2}-z_{1}^{2}\right|^{4}\left[\left(|w|^{2}-1\right)^{2}+2|u|^{2}|| w\left|-\frac{\beta^{2}-z_{1}^{2}}{\left|\beta^{2}-z_{1}^{2}\right|}\right|^{2}\right] \\
& =\left|\beta^{2}-z_{1}^{2}\right|^{4}\left[\left(|w|^{2}-1\right)^{2}+2|u|^{2}\left(|w|^{2}+1-2|u|^{2} \Re \frac{w}{u^{2}}\right)\right] \\
& =\left|\beta^{2}-z_{1}^{2}\right|^{4}\left[\left(|w|^{2}-1\right)^{2}+2\left(|u|^{2}|w|^{2}+|u|^{2}-2 \Re\left(w \bar{u}^{2}\right)\right)\right] .
\end{aligned}
$$


If we denote by

$$
\begin{aligned}
J(u, w) & =1+|u|^{2}+|w|^{2}, \\
H(u, w) & =\left(|w|^{2}-1\right)^{2}+2\left(|u|^{2}|w|^{2}+|u|^{2}-2 \Re\left(w \bar{u}^{2}\right)\right),
\end{aligned}
$$

we see using Lemma 2.5 and the above formula for $M(z)$ that

$$
g^{\star}(z)=\log \left|\frac{\beta^{2}-z_{1}^{2}}{1-\beta^{2} z_{1}^{2}}\right|+\log \left[\left(\frac{J+\sqrt{H}}{2}\right)^{1 / 2}(F(z))\right] .
$$

We finally note that $J^{2}-H=\left|u^{2}+2 w\right|^{2}$, so

$$
\left(\frac{J+\sqrt{H}}{2}\right)^{1 / 2}=\frac{1}{2}\left(\sqrt{J+\left|u^{2}+2 w\right|}+\sqrt{J-\left|u^{2}+2 w\right|}\right),
$$

and the proof of the lemma is finished.

Lemma 2.7. The function $V$ defined by (2.18) is real analytic, plurisubharmonic and maximal in $\mathbb{C} \times \Delta$.

Proof. The fact that $V$ is real analytic follows if we write

$$
V(u, w)=\frac{1}{2} \log \left[2\left(1+|u|^{2}+|w|^{2}\right)+2 \sqrt{\left(1+|u|^{2}+|w|^{2}\right)^{2}-\left|u^{2}+2 w\right|^{2}}\right]
$$

and notice that $1+|u|^{2}+|w|^{2}-\left|u^{2}+2 w\right| \geq(1-|w|)^{2}>0$ on $\mathbb{C} \times \Delta$. We compute the Levi form of $V$. Setting

$$
\begin{aligned}
G_{1} & =G_{1}(u, w)=1+|u|^{2}+|w|^{2}+\left|u^{2}+2 w\right|, \\
G_{2} & =G_{2}(u, w)=1+|u|^{2}+|w|^{2}-\left|u^{2}+2 w\right|, \\
G & =G(u, w)=\sqrt{G_{1}}+\sqrt{G_{2}},
\end{aligned}
$$

we have $V=\log G$. We obtain (see $[\mathbf{C}]$ for the details of the computations):

$$
\langle(L V) t, t\rangle=\frac{1}{2\left(G_{1} G_{2}\right)^{3 / 2}}\left|\left(1-|w|^{2}\right) t_{1}+(u \bar{w}-\bar{u}) t_{2}\right|^{2}
$$

at any point $(u, w) \in \mathbb{C} \times \Delta$, where $t=\left(t_{1}, t_{2}\right) \in \mathbb{C}^{2}$. So $V$ is plurisubharmonic in $\mathbb{C} \times \Delta$. Moreover, since

$$
\frac{\partial^{2} V}{\partial u \partial \bar{u}} \frac{\partial^{2} V}{\partial w \partial \bar{w}}-\frac{\partial^{2} V}{\partial u \partial \bar{w}} \frac{\partial^{2} V}{\partial w \partial \bar{u}} \equiv 0
$$

we see that $V$ is maximal.

As an immediate consequence of Lemma 2.6 and Lemma 2.7 we have the following:

Corollary 2.8. The function $g^{\star}$ is real analytic, plurisubharmonic and maximal in $D$.

Lemma 2.9. $g^{\star} \in C^{1,1}\left(B^{2} \backslash\{p, q\}\right)$. 
Proof. Let $v$ be the function defined on $D$ by $v(z)=g^{\star}(z), z \in D$ (i.e., $v$ is given by the formula in the statement of Lemma 2.5). We first notice that there exists a domain $D^{\prime} \subset B^{2} \backslash\{p, q\}$ such that $\left(\bar{D} \cap B^{2}\right) \backslash\{p, q\} \subset D^{\prime}$ and the function $v$ is well defined, real analytic and plurisubharmonic on $D^{\prime}$. Indeed, by Lemma 2.6 and Lemma $2.7 v$ has the above mentioned properties near all points $z \in B^{2} \backslash\{p, q\}$ for which $|w(z)|<1$, where $w(z)$ is given by (2.17). So it is enough to check that $|w(z)|<1$ for $z \in \partial D \cap\left(B^{2} \backslash\{p, q\}\right)$. Without loss of generality we assume that $\beta z_{2}=\eta\left(\beta-z_{1}\right)$, where $|\eta|=1$. Then $z_{1}=\beta-\beta z_{2} / \eta$ satisfies $\Re z_{1}>0$, hence

$$
|w(z)|=\left|\frac{\beta^{2} z_{2}^{2}}{\beta^{2}-z_{1}^{2}}\right|=\left|\frac{\beta-z_{1}}{\beta+z_{1}}\right|<1 .
$$

We recall that the function $g_{2}(\cdot, p)$ is real analytic on $B^{2} \backslash\{p, q\}$. So in order to prove that $g^{\star} \in C^{1,1}\left(B^{2} \backslash\{p, q\}\right)$ it is enough, by symmetry reasons, to show that the function $v-g_{2}(\cdot, p)$ vanishes to first order at points $z_{0}=\left(z_{1}^{0}, z_{2}^{0}\right) \in B^{2} \backslash\{p\}$ of the form $\beta-z_{1}^{0}=\eta_{0} \beta z_{2}^{0}$ with $\left|\eta_{0}\right|=1$. Near such a point $z_{0}$ we make the change of variables

$$
\left(z_{1}, z_{2}\right) \rightarrow\left(\eta, z_{2}\right), \quad \eta=\frac{\beta-z_{1}}{\beta z_{2}}
$$

Using these coordinates we obtain after a straightforward computation:

$$
g_{2}(z, p)=\frac{1}{2} \log \frac{\left|z_{2}\right|^{2}}{\left|1-\beta^{2} z_{1}^{2}\right|^{2}}+\frac{1}{2} \log h_{1}\left(\eta, z_{2}\right),
$$

where

$$
h_{1}\left(\eta, z_{2}\right)=\left(1-\beta^{2}+\beta^{2}|\eta|^{2}\right)\left|1+\beta^{2}\left(1-\eta z_{2}\right)\right|^{2},
$$

and

$$
v(z)=\frac{1}{2} \log \frac{\left|z_{2}\right|^{2}}{\left|1-\beta^{2} z_{1}^{2}\right|^{2}}+\frac{1}{2} \log h_{2}\left(\eta, z_{2}\right),
$$

where

$$
\begin{aligned}
h_{2}\left(\eta, z_{2}\right) & =\frac{\beta^{4}|\eta|^{2}\left|\eta z_{2}-2\right|^{2}+\beta^{4}\left|z_{2}\right|^{2}+2\left(1-\beta^{4}\right)+\beta^{2} \sqrt{\widetilde{M}}}{2} \\
\widetilde{M} & =\beta^{4}\left(\left|z_{2}\right|^{2}-|\eta|^{2}\left|\eta z_{2}-2\right|^{2}\right)^{2}+4\left(1-\beta^{4}\right)\left|\bar{z}_{2}+\eta^{2} z_{2}-2 \eta\right|^{2} .
\end{aligned}
$$

Hence it is enough to check that at the point $\left(\eta_{0}, z_{2}^{0}\right)$ we have $h_{1}=h_{2}$, $\partial h_{1} / \partial z_{2}=\partial h_{2} / \partial z_{2}$, and $\partial h_{1} / \partial \eta=\partial h_{2} / \partial \eta$, where $\left|\eta_{0}\right|=1$ and $\left|z_{2}^{0}\right|<1$. A simple computation shows

$$
\widetilde{M}\left(\eta_{0}, z_{2}\right)=16\left[1-\Re\left(\eta_{0} z_{2}\right)\right]^{2},
$$

hence

$$
h_{1}\left(\eta_{0}, z_{2}\right)=h_{2}\left(\eta_{0}, z_{2}\right)=\left|1+\beta^{2}-\beta^{2} \eta_{0} z_{2}\right|^{2},
$$


for all $\left|z_{2}\right|<1$. This proves the first two of the above relations. For the third one we obtain after a computation that

$$
\begin{aligned}
& \frac{\partial \widetilde{M}}{\partial \eta}\left(\eta_{0}, z_{2}\right)=16\left[\Re\left(\eta_{0} z_{2}\right)-1\right]\left(z_{2}-\bar{\eta}_{0}\right)\left[1-\beta^{4}-\beta^{4}\left(\bar{\eta}_{0} \bar{z}_{2}-2\right)\right], \\
& \frac{\partial h_{1}}{\partial \eta}\left(\eta_{0}, z_{2}\right)=\frac{\partial h_{2}}{\partial \eta}\left(\eta_{0}, z_{2}\right)=\beta^{2}\left(1+\beta^{2}\right)\left(z_{2}-\bar{\eta}_{0}\right)\left(\beta^{2} \bar{\eta}_{0} \bar{z}_{2}-1-\beta^{2}\right),
\end{aligned}
$$

and the proof is finished.

Remark. The function $g^{\star}$ is not of class $C^{2}$. Indeed, with the notations introduced in the preceding proof one can check that $\frac{\partial^{2} h_{1}}{\partial \eta \partial \bar{\eta}}\left(\eta_{0}, z_{2}\right) \neq$ $\frac{\partial^{2} h_{2}}{\partial \eta \partial \bar{\eta}}\left(\eta_{0}, z_{2}\right)$.

Proposition 2.10. The function $g^{\star}$ is negative and plurisubharmonic on $B^{2}$, and it has logarithmic poles with weight one at $p$ and $q$.

Proof. By the construction of $g^{\star}$ we clearly have $g^{\star}<0$ on $B^{2}$. It follows by inspection that $g^{\star}$ has logarithmic poles with weight one at $p$ and $q$. We have shown (Corollary 2.8) that $g^{\star}$ is plurisubharmonic on int $\Gamma_{p} \cup D \cup \operatorname{int} \Gamma_{q}$. Let $v$ and $D^{\prime}$ be as defined in the proof of Lemma 2.9. We recall that $\left(\bar{D} \cap B^{2}\right) \backslash$ $\{p, q\} \subset D^{\prime}$ and that the function $v$ is real analytic and plurisubharmonic on $D^{\prime}$. In order to show that $g^{\star}$ is plurisubharmonic on $B^{2}$ we consider a point $z_{0} \in B^{2} \cap \partial D$ and a complex line $L$ through $z_{0}$. Without loss of generality we assume $z_{0} \in \partial \Gamma_{p}$. If $L \cap B^{2} \subset \partial \Gamma_{p}$ then $\left.g^{\star}\right|_{L}$ is subharmonic, since $g^{\star}=g_{2}(\cdot, p)$ on $\Gamma_{p}$. Otherwise we apply Lemma 2.11 to conclude that $\left.g^{\star}\right|_{L}$ is subharmonic near $z_{0}$.

Lemma 2.11. Let $\Gamma$ be an embedded smooth curve in $\mathbb{C}$ which divides $\mathbb{C}$ into two domains $\Gamma_{+}$and $\Gamma_{-}$. Let $\Omega$ be a disc, $\Omega_{+}=\Omega \cap \Gamma_{+}, \Omega_{-}=\Omega \cap \Gamma_{-}$. Assume $v_{+}$and $v_{-}$are subharmonic functions of class $C^{2}$, defined in a neighborhood of $\bar{\Omega}_{+}$, respectively $\bar{\Omega}_{-}$, such that $v_{+}-v_{-}$vanishes to first order along $\Gamma$. Then the function $v$ defined by $v=v_{+}$on $\bar{\Omega}_{+}, v=v_{-}$on $\bar{\Omega}_{-}$, is subharmonic in $\Omega$.

Proof. It suffices to show that $\Delta v \geq 0$ in the sense of distributions. Let $n_{+}$ (respectively $n_{-}$) denote the unit outward normal vector of $\Gamma$ with respect to $\Omega_{+}$(respectively $\Omega_{-}$). We let $\phi \in C_{0}^{\infty}(\Omega), \phi \geq 0$, and apply Green's formula on $\Omega_{+}$and $\Omega_{-}$using the fact that $v_{+}=v_{-}$to first order on $\Gamma$. We get

$$
\begin{aligned}
\int_{\Omega_{+}} v_{+} \Delta \phi-\phi \Delta v_{+} & =\int_{\Gamma} v_{+} \frac{\partial \phi}{\partial n_{+}}-\phi \frac{\partial v_{+}}{\partial n_{+}} \\
& =-\int_{\Gamma} v_{-} \frac{\partial \phi}{\partial n_{-}}-\phi \frac{\partial v_{-}}{\partial n_{-}}=-\int_{\Omega_{-}} v_{-} \Delta \phi-\phi \Delta v_{-}
\end{aligned}
$$


hence

$$
\int_{\Omega} v \Delta \phi=\int_{\Omega_{+}} \phi \Delta v_{+}+\int_{\Omega_{-}} \phi \Delta v_{-} \geq 0
$$

The proof of Theorem 2 is complete as soon as we show the following:

Proposition 2.12. The partial derivatives $\frac{\partial}{\partial z_{1}} g_{2}(\cdot, p, q)$ and $\frac{\partial}{\partial z_{2}} g_{2}(\cdot, p, q)$ extend continuously to $\partial B^{2}$.

Proof. If $z_{0} \in \partial B^{2} \backslash \bar{D}$ then the function $g_{2}(\cdot, p, q)$ is clearly defined and real analytic in a neighborhood of $z_{0}$. By Lemmas 2.6, 2.7 and 2.9 the function $g_{2}(\cdot, p, q)$ is well defined and of class $C^{1}$ in a neighborhood of any point $z_{0} \in \partial B^{2} \cap \bar{D}$ such that $\left|w\left(z_{0}\right)\right|<1$, where $|w(z)|<1$ is defined by (2.17). So we only have to consider the points $z_{0} \in \partial B^{2} \cap \bar{D}$ where $\left|w\left(z_{0}\right)\right|=1$. At such points $z_{0}=\left(z_{1}^{0}, z_{2}^{0}\right)$ we have $\beta\left|z_{2}^{0}\right|=\left|\beta-z_{1}^{0}\right|=\left|\beta+z_{1}^{0}\right|$, so $z_{1}^{0}=0$ and $\left|z_{2}^{0}\right|=1$. Let us fix $z_{0}=(0, \gamma) \in \partial B^{2}$. Let $v$ be the function defined as in the proof of Lemma 2.9 by $v(z)=g_{2}(z, p, q)$, for $z \in D$. Since

$$
\begin{aligned}
{\left[\frac{\partial}{\partial z_{1}} g_{2}(\cdot, p)\right]\left(z_{0}\right) } & =\left[\frac{\partial}{\partial z_{1}} g_{2}(\cdot, q)\right]\left(z_{0}\right)=0 \\
{\left[\frac{\partial}{\partial z_{2}} g_{2}(\cdot, p)\right]\left(z_{0}\right) } & =\left[\frac{\partial}{\partial z_{2}} g_{2}(\cdot, q)\right]\left(z_{0}\right)=\frac{1}{2}\left(1-\beta^{2}\right) \bar{\gamma}
\end{aligned}
$$

it suffices to show that

$$
\lim _{z \rightarrow z_{0}} \frac{\partial v}{\partial z_{1}}(z)=0 \text { and } \lim _{z \rightarrow z_{0}} \frac{\partial v}{\partial z_{2}}(z)=\frac{1}{2}\left(1-\beta^{2}\right) \bar{\gamma} .
$$

Here, as well as in the remainder of this proof, the notation $\lim _{z \rightarrow z_{0}}$ means that $z \in \bar{D} \cap B^{2}$ and $z \rightarrow z_{0}$. If $M(z)$ is defined as in the statement of Theorem 2 then it follows from the definition of $v$ that

$$
\begin{aligned}
\lim _{z \rightarrow z_{0}} \frac{\partial v}{\partial z_{1}}(z) & =\frac{1}{8} \lim _{z \rightarrow z_{0}}\left[(M(z))^{-1 / 2} \frac{\partial M}{\partial z_{1}}(z)\right], \\
\lim _{z \rightarrow z_{0}} \frac{\partial v}{\partial z_{2}}(z) & =\frac{\bar{\gamma}}{2}+\frac{1}{8} \lim _{z \rightarrow z_{0}}\left[(M(z))^{-1 / 2} \frac{\partial M}{\partial z_{2}}(z)\right] .
\end{aligned}
$$

For $z \in \bar{D} \cap B^{2}$ let us introduce the following notations:

$$
\begin{aligned}
E_{1}=E_{1}(z) & =\frac{\left.\left|\beta^{2}\right| z_{2}\right|^{2}-\left(\beta^{2}-z_{1}^{2}\right) \mid}{\left|\beta^{2}-z_{1}^{2}\right|-\beta^{2}\left|z_{2}\right|^{2}}, \\
E_{2}=E_{2}(z) & =\frac{\beta^{2}\left|z_{2}\right|^{2}-\Re\left(\beta^{2}-z_{1}^{2}\right)}{\beta^{2}\left|z_{2}\right|^{2}-\left|\beta^{2}-z_{1}^{2}\right|}, \\
F=F(z) & =\frac{\left|\beta^{2}-z_{1}^{2}\right|-\Re\left(\beta^{2}-z_{1}^{2}\right)}{\left(\left|\beta^{2}-z_{1}^{2}\right|-\beta^{2}\left|z_{2}\right|^{2}\right)^{2}} .
\end{aligned}
$$


We will prove (Lemma 2.13) that $\lim _{z \rightarrow z_{0}} F(z)=0$. Since $E_{1}^{2}-1=$ $2 \beta^{2}\left|z_{2}\right|^{2} F$ and $E_{2}-1=\left(\beta^{2}\left|z_{2}\right|^{2}-\left|\beta^{2}-z_{1}^{2}\right|\right) F$ it follows that $\lim _{z \rightarrow z_{0}} E_{1}(z)=$ $\lim _{z \rightarrow z_{0}} E_{2}(z)=1$.

A simple computation now shows:

$$
\begin{aligned}
& \frac{\partial M / \partial z_{2}(z)}{\beta^{2}\left|z_{2}\right|^{2}-\left|\beta^{2}-z_{1}^{2}\right|} \\
& =4 \beta^{2}\left|z_{2}\right|^{2} \bar{z}_{2}\left[\beta^{2}\left(\beta^{2}\left|z_{2}\right|^{2}+\left|\beta^{2}-z_{1}^{2}\right|\right)+2\left(1-\beta^{4}\right) E_{2}\right] \\
& \quad-\left.4\left(1-\beta^{4}\right) \bar{z}_{2}\left|\beta^{2}\right| z_{2}\right|^{2}-\left(\beta^{2}-z_{1}^{2}\right) \mid E_{1} \rightarrow 8 \beta^{2} \bar{\gamma}, \\
& \quad \frac{\left|\partial M / \partial z_{1}(z)\right|}{\left|\beta^{2}-z_{1}^{2}\right|-\beta^{2}\left|z_{2}\right|^{2}} \\
& \leq 4\left|z_{1}\right|\left[\left|\beta^{2}-z_{1}^{2}\right|\left(\beta^{2}\left|z_{2}\right|^{2}+\left|\beta^{2}-z_{1}^{2}\right|\right)+2\left(1-\beta^{4}\right)\left|z_{2}\right|^{2} E_{1}\right] \rightarrow 0, \\
& \quad \sqrt{M(z)} \\
& \left|\beta^{2}-z_{1}^{2}\right|-\beta^{2}\left|z_{2}\right|^{2} \\
& =\sqrt{\left(\beta^{2}\left|z_{2}\right|^{2}+\left|\beta^{2}-z_{1}^{2}\right|\right)^{2}+4\left(1-\beta^{4}\right)\left|z_{2}\right|^{2} E_{1}^{2}} \rightarrow 2,
\end{aligned}
$$

as $z \in \bar{D} \cap B^{2} \rightarrow z_{0}=(0, \gamma)$. These formulas, together with relations $(2.20)$ and (2.21), now show that (2.19) is verified.

Lemma 2.13. If $F(z)$ is defined by (2.22) for $z \in\left(\bar{D} \cap B^{2}\right) \backslash\{p, q\}$ then $F(z) \rightarrow 0$ as $z \rightarrow z_{0}=(0, \gamma) \in \partial B^{2}$.

Proof. Let us define

$$
F_{1}(z)=\frac{\left|\beta^{2}-z_{1}^{2}\right|+\Re\left(\beta^{2}-z_{1}^{2}\right)}{\left(\left|\beta^{2}-z_{1}^{2}\right|+\beta^{2}\left|z_{2}\right|^{2}\right)^{2}} F(z)=\frac{\left(\Im z_{1}^{2}\right)^{2}}{\left(\left|\beta^{2}-z_{1}^{2}\right|^{2}-\beta^{4}\left|z_{2}\right|^{4}\right)^{2}} .
$$

It is enough to show $F_{1}(z) \rightarrow 0$ as $z \rightarrow z_{0}$. We claim that for $z \in \bar{D} \cap B^{2}$ we have

$$
\left|\beta^{2}-z_{1}^{2}\right|^{2}-\beta^{4}\left|z_{2}\right|^{4} \geq \beta^{2}\left|z_{2}\right|^{2}\left(\left|z_{1}\right|^{2}+2 \beta|x|\right),
$$

where $z_{1}=x+i y$. Indeed, let us assume without loss of generality that $x \geq 0$. Then since $z \in \bar{D}$ we have $\left|\beta-z_{1}\right| \geq \beta\left|z_{2}\right|$, so

$$
\begin{aligned}
& \left|\beta^{2}-z_{1}^{2}\right|^{2}-\beta^{4}\left|z_{2}\right|^{4} \geq \beta^{2}\left|z_{2}\right|^{2}\left(\left|\beta+z_{1}\right|^{2}-\beta^{2}\left|z_{2}\right|^{2}\right)= \\
& =\beta^{2}\left|z_{2}\right|^{2}\left(\beta^{2}\left(1-\left|z_{2}\right|^{2}\right)+\left|z_{1}\right|^{2}+2 \beta x\right) \geq \beta^{2}\left|z_{2}\right|^{2}\left(\left|z_{1}\right|^{2}+2 \beta x\right) .
\end{aligned}
$$

It follows that

$$
\begin{aligned}
F_{1}(z) & \leq \frac{1}{\beta^{4}\left|z_{2}\right|^{4}} \frac{\left(\Im z_{1}^{2}\right)^{2}}{\left(\left|z_{1}\right|^{2}+2 \beta|x|\right)^{2}}=\frac{4}{\beta^{4}\left|z_{2}\right|^{4}} \frac{x^{2} y^{2}}{\left(x^{2}+y^{2}+2 \beta|x|\right)^{2}} \\
& \leq \frac{4}{\beta^{4}\left|z_{2}\right|^{4}} \frac{y^{2}}{(2 \beta+|x|)^{2}} \rightarrow 0,
\end{aligned}
$$

as $z=\left(x+i y, z_{2}\right) \rightarrow(0, \gamma) \in \partial B^{2}$. 
Proof of Corollary 3. Let $z=\left(z_{1}, z^{\prime}\right) \in B^{n}$. If $z^{\prime}=0$ then $g_{n}(z, p, q)=$ $g_{n}(z, p)+g_{n}(z, q)$ verifies the desired formula. So we assume $z^{\prime} \neq 0$ and let $u=\left(0, z^{\prime} /\left\|z^{\prime}\right\|\right)$. We denote by $B^{2}$ the unit ball of the subspace $V_{u}=$ $\mathbb{C} e_{1}+\mathbb{C} u$ and consider the inclusion map $j: B^{2} \rightarrow B^{n}$ and the orthogonal projection $\pi: B^{n} \rightarrow B^{2}$. As $z=z_{1} e_{1}+\left\|z^{\prime}\right\| u$ we get the desired formula by using the fact that pluricomplex Green functions are decreasing with respect to holomorphic mappings. The rest of the assertions of Corollary 3 now follow easily from Theorem 2.

\section{Proofs of Proposition 4 and Theorem 5.}

In this section we consider the case when the poles have different weights. Let us recall from Section 1 that $A=\{(p, \mu),(q, \nu)\} \subset B^{n} \times(0,+\infty), \mu \geq \nu$, and that $g_{n}(\cdot, A)$ denotes the pluricomplex Green function of $B^{n}$ with poles in $A$. We saw that without loss of generality we can assume $p=0$ and $q=(\alpha, 0, \ldots, 0)$, where $\alpha \in(0,1)$. We also recall the following notations: $L_{u}=\{\zeta u: \zeta \in \Delta\}$, where $u=\left(u_{1}, \ldots, u_{n}\right) \in \partial B^{n}, \Gamma_{0}=\cup\left\{L_{u}:\left|u_{1}\right| \leq\right.$ $\alpha / 2\}$, and $\Gamma_{q}=T_{q}\left(\Gamma_{0}\right)$, where $T_{q} \in \operatorname{Aut}\left(B^{n}\right)$ is given by (2.1).

Proof of Proposition 4. For the proof of (1.7) let us fix $u \in \partial B^{n}$ such that $\left|u_{1}\right| \leq \alpha / 2$. We construct a holomorphic map $F: B^{n} \rightarrow \Delta$ such that $F(q)=0$ and $F(\zeta u)=\zeta$ for all $\zeta \in \Delta$ (see [R], p. 164). We choose $u^{2}, \ldots, u^{n} \in \partial B^{n}$ such that $\left\{u, u^{2}, \ldots, u^{n}\right\}$ is an orthonormal basis of $\mathbb{C}^{n}$. Then $\|z\|^{2}=|\langle z, u\rangle|^{2}+\left|\left\langle z, u^{2}\right\rangle\right|^{2}+\cdots+\left|\left\langle z, u^{n}\right\rangle\right|^{2}$, for all $z \in \mathbb{C}^{n}$; here $\langle\cdot, \cdot \cdot\rangle$ denotes the standard scalar product on $\mathbb{C}^{n}$. Let $h(\zeta)=1-\sqrt{1-\zeta}$, for $\zeta \in \Delta$. Then $h$ is holomorphic in $\Delta$ and the Taylor expansion of $h$ at 0 has positive coefficients; hence $|h(\zeta)| \leq h(|\zeta|)$, for all $\zeta \in \Delta$. For $a \in \mathbb{C}$ with $|a| \leq 1$ and for $\theta_{2}, \ldots, \theta_{n} \in \mathbb{R}$ we define $F: B^{n} \rightarrow \mathbb{C}$ by

$$
F(z)=\langle z, u\rangle+a h\left(\sum_{j=2}^{n} e^{i \theta_{j}}\left\langle z, u^{j}\right\rangle^{2}\right) .
$$

Since $|a| \leq 1$ we have by the properties of $h$ that

$$
|F(z)| \leq|\langle z, u\rangle|+h\left(\sum_{j=2}^{n}\left|\left\langle z, u^{j}\right\rangle\right|^{2}\right)<|\langle z, u\rangle|+h\left(1-|\langle z, u\rangle|^{2}\right)=1
$$

here we also used the fact that $h$ is increasing on $[0,1]$. Hence $F\left(B^{n}\right) \subseteq \Delta$ and clearly $F(\zeta u)=\zeta$ for all $\zeta \in \Delta$.

We now show that since $u$ satisfies $\left|u_{1}\right| \leq \alpha / 2$ we can choose $a$ with $|a| \leq 1$ and $\theta_{2}, \ldots, \theta_{n} \in \mathbb{R}$ such that $F(q)=0$. For $j=2, \ldots, n$ we choose $\theta_{j}$ such 
that $e^{i \theta_{j}}\left\langle q, u^{j}\right\rangle^{2}=\left|\left\langle q, u^{j}\right\rangle\right|^{2}$. Next we define $a$ by

$$
a=-\frac{\langle q, u\rangle}{h\left(\sum_{j=2}^{n}\left|\left\langle q, u^{j}\right\rangle\right|^{2}\right)} .
$$

Then clearly $F(q)=0$, so we only have to check that $|a| \leq 1$. But this is equivalent to

$$
\left(1-\sum_{j=2}^{n}\left|\left\langle q, u^{j}\right\rangle\right|^{2}\right)^{\frac{1}{2}} \leq 1-|\langle q, u\rangle|,
$$

which in turn is equivalent to

$$
\alpha^{2}=|\langle q, u\rangle|^{2}+\sum_{j=2}^{n}\left|\left\langle q, u^{j}\right\rangle\right|^{2} \geq 2|\langle q, u\rangle|=2 \alpha\left|u_{1}\right| .
$$

Using the function $F$ constructed above it follows from the definition of $g_{n}(\cdot, A)$ that $\mu \log |F(z)| \leq g_{n}(z, A)$, for all $z \in B^{n}$. On the other hand we clearly have $g_{n}(z, A) \leq \mu g_{n}(z, 0)=\mu \log \|z\|$. So for $z=\zeta u$ we obtain

$$
\mu \log |\zeta|=\mu \log |F(\zeta u)| \leq g_{n}(\zeta u, A) \leq \mu g_{n}(\zeta u, 0)=\mu \log |\zeta|,
$$

so (1.7) holds for all $z \in \Gamma_{0}$.

The proof of (1.8) is done in a similar way. We first interchange $p=0$ and $q$ by applying $T_{q}$, so the pole of smaller weight is now at the origin. Then the lower bound in (1.8) is obtained exactly as before, and the upper bound actually holds for all $z \in B^{n}$.

Since for $z$ on the $z_{1}$-axis we have $g_{n}(z, A)=\mu g_{n}(z, p)+\nu g_{n}(z, q)<$ $\mu g_{n}(z, p),(1.7)$ implies that $g_{n}(\cdot, A)$ cannot be real analytic on $B^{n} \backslash\{p, q\}$. The last assertion of Proposition 4 follows from the next lemma.

In the above setting we consider the case when $p=0, \mu=1$, and $q=$ $(\alpha, 0, \ldots, 0), \nu=1 / 2$, with $\alpha \in(0,1)$ arbitrary. For $u=\left(u_{1}, \ldots, u_{n}\right) \in \partial B^{n}$ we let

$$
\widetilde{\Gamma}_{0}=\bigcup\left\{L_{u}:\left|u_{1}\right|^{2} \leq 1 / 2\right\}
$$

Lemma 3.1. For any $\alpha \in(0,1)$ and $z \in \widetilde{\Gamma}_{0}$ we have $g_{n}(z, A)=g_{n}(z, 0)=$ $\log \|z\|$. If $\alpha^{2}<1 / 2$ then there is no complex line $L$ containing $q$ and such that $g_{n}(z, A)=g_{n}(z, q) / 2$ along $L \cap B^{n}$.

Proof. We fix $u \in \partial B^{n}$ with $\left|u_{1}\right|^{2} \leq 1 / 2$ and choose $u^{2}, \ldots, u^{n} \in \partial B^{n}$ such that $\left\{u, u^{2}, \ldots, u^{n}\right\}$ is an orthonormal basis of $\mathbb{C}^{n}$. For $a \in \mathbb{C}$ with $|a| \leq 1$ and for $\theta_{2}, \ldots, \theta_{n} \in \mathbb{R}$ we consider the function $F: B^{n} \rightarrow \Delta$ defined by

$$
F(z)=\langle z, u\rangle^{2}+a \sum_{j=2}^{n} e^{i \theta_{j}}\left\langle z, u^{j}\right\rangle^{2} .
$$


For $j=2, \ldots, n$ we choose $\theta_{j}$ such that $e^{i \theta_{j}}\left\langle q, u^{j}\right\rangle^{2}=\left|\left\langle q, u^{j}\right\rangle\right|^{2}$ and then we choose $a \in \mathbb{C}$ such that $F(q)=0$. We note that $\left|u_{1}\right|^{2} \leq 1 / 2$ implies $|a| \leq 1$. Since $F(\zeta u)=\zeta^{2}$ and $F(q)=0$, we get by the definition of $g_{n}(z, A)$ that

$$
\frac{1}{2} \log |F(z)| \leq g_{n}(z, A) \leq \log \|z\|,
$$

hence $g_{n}(z, A)=\log \|z\|$ for $z=\zeta u$.

We now assume that $\alpha^{2}<1 / 2$ and that $L$ is a complex line containing $q$, different from the $z_{1}$-axis. We parametrize $L \cap B^{n}$ using the unit disc $\Delta$; for instance $L \cap B^{n}=f(\Delta)$, where $f(\zeta)=T_{q}\left(\zeta u^{\star}\right)$ for a suitable $u^{\star} \in \partial B^{n}$. Since $\alpha^{2}<1 / 2$ there is a nonempty open set $G \subset \Delta$ such that $f(G) \subset \widetilde{\Gamma}_{0}$. For $z \in G$ we have $g_{n}(z, A)=\log \|z\| \neq g_{n}(z, q) / 2$.

Remark. In the above setting let $S=\left\{z \in B^{n} \backslash\{q\}: g_{n}(z, A)=\right.$ $\left.g_{n}(z, q) / 2\right\}$. If $\alpha^{2}<1 / 2$ we have in fact $S=\emptyset$. Indeed, if $z \in S$ and $L$ is the complex line through $q$ and $z$ then by the maximum principle it follows that $L \cap B^{n} \subset S$, which contradicts Lemma 3.1.

Remark. Let us recall the description of the foliation corresponding to $g_{n}(\cdot, p, q)$ given in Corollary 3: The leaves are embedded submanifolds of $B^{n}$, some passing through $p$ and not containing $q$, or through $q$ and not containing $p$, and some passing through both $p$ and $q$. In the case of different weights $\mu=1, \nu=1 / 2$, and when $\alpha^{2}<1 / 2$, Lemma 3.1 shows that there are no "nice" leaves passing through $q$ and not through $p$. Indeed, let us assume that the function $f: \Delta \rightarrow B^{n}$ is proper holomorphic, that there is a unique $s \in \Delta$ with $f(s)=q$, and that $f^{\prime}(s) \neq 0$. If the function $\zeta \rightarrow g_{n}(f(\zeta), A)$ is harmonic on $\Delta \backslash\{s\}$, it follows from the maximum principle that $g_{n}(f(\zeta), q) / 2 \leq g_{n}(f(\zeta), A)$, hence these functions are equal in $\Delta$. This implies that $f(\Delta)$ is a complex line, which is in contradiction to Lemma 3.1.

Proof of Theorem 5. It is clear that if such a sequence $\left\{F_{j}\right\}_{j}$ exists then $g_{n}\left(z_{0}, A\right)=\mu \log |\zeta|=\mu g_{n}\left(z_{0}, 0\right)$. Conversely, we assume that $\mu g_{n}\left(z_{0}, 0\right)=$ $g_{n}\left(z_{0}, A\right)$. We first note that $g_{n}(t u, A)=\mu g_{n}(t u, 0)$ for all $t \in \Delta$. Indeed, the function $v(t)=g_{n}(t u, A)-\mu g_{n}(t u, 0)$ is subharmonic in $\Delta \backslash\{0\}$. Since $v \leq 0$ on $\Delta \backslash\{0\}$ and since, by hypothesis, $v(\zeta)=0$, it follows from the maximum principle that $v \equiv 0$.

Let $L_{u}=\{t u: t \in \Delta\}$ and let $F: B^{n} \rightarrow \Delta, F(z)=\langle z, u\rangle$. We consider the following Hartogs domains in $\mathbb{C}^{n+1}$ :

$$
\begin{gathered}
D_{1}=\left\{(z, w) \in B^{n} \times \mathbb{C}:|w|<e^{-g_{n}(z, A) / \mu}\right\}, \\
D_{2}=\left\{(z, w) \in B^{n} \times \mathbb{C}:|w|<e^{-g_{n}(z, 0)}\right\} .
\end{gathered}
$$

The domains $D_{1}$ and $D_{2}$ are pseudoconvex (see $\left.[\mathbf{B r}]\right)$. Let $u=\left(u_{1}, \ldots, u_{n}\right)$ and write $z=\left(z_{1}, \ldots, z_{n}\right)$. We consider the holomorphic functions $h_{j}(z)=$ 
$z_{j}-\langle z, u\rangle u_{j}, j=1, \ldots, n$, and we denote by $X$ the analytic variety defined by these functions in $B^{n} \times \mathbb{C}$ :

$$
X=\left\{(z, w) \in B^{n} \times \mathbb{C}: h_{1}(z)=\cdots=h_{n}(z)=0\right\}=L_{u} \times \mathbb{C} .
$$

As $\log |F(z)| \leq g_{n}(z, 0)$, the function $\alpha(z, w)=1 /(1-F(z) w)$ is holomorphic in $D_{2}$. Since $g_{n}(t u, A)=\mu g_{n}(t u, 0)$ we have that $X \cap D_{1}=X \cap D_{2}$. As the variety $X \cap D_{1}$ is globally defined in $D_{1}$ and $\alpha$ is holomorphic in a neighborhood of $X \cap D_{1}$, it is a standard result that there exists a holomorphic function $\widetilde{\alpha}$ on $D_{1}$ such that $\widetilde{\alpha}=\alpha$ on $X \cap D_{1}$ ([H2], Theorem 4.2.12). We can easily adapt the proof of the above quoted theorem in order to ensure that our extension $\widetilde{\alpha}$ also satisfies $\widetilde{\alpha}(q, w)=0$, for all $w \in \mathbb{C}$. This can be done as follows: Let $\chi \in C^{\infty}\left(D_{1}\right)$ be such that $\chi \equiv 1$ in a neighborhood of $X \cap D_{1}$ (relatively to $D_{1}$ ), supp $\chi \subset D_{2}$, and $\{(q, w): w \in \mathbb{C}\} \subset D_{1} \backslash \operatorname{supp} \chi$. Let $\phi(z, w)=\frac{1}{2} \log \left(\left|h_{1}(z)\right|^{2}+\cdots+\left|h_{n}(z)\right|^{2}\right)+\log \|z-q\|$, for $(z, w) \in D_{1}$. We consider the $\bar{\partial}$-closed $(0,1)$ form $\bar{\partial}(\chi \alpha)=\alpha \bar{\partial} \chi$ and we solve $\bar{\partial} U=\alpha \bar{\partial} \chi$ with the following $L^{2}$ estimate ([H1], Theorem 4.4.2):

$$
\int_{D_{1}}|U|^{2} e^{-2(n+1)(\phi+\psi)}(1+\|(z, w)\|)^{-2} d \lambda \leq \int_{D_{1}}|\alpha \bar{\partial} \chi|^{2} e^{-2(n+1)(\phi+\psi)} d \lambda .
$$

Here $\psi$ is a plurisubharmonic exhaustion function for $D_{1}$ increasing rapidly to $\infty$, so that the right hand side of the above inequality is finite (this is possible since the function $|\alpha \bar{\partial} \chi|^{2} e^{-2(n+1)(\phi+\psi)}$ is continuous on $\left.D_{1}\right)$. Since the function $e^{-2(n+1)(\phi+\psi)}$ is not integrable near any point of $X \cap D_{1}$ and near any point of the form $(q, w), w \in \mathbb{C}$, it follows that $U$ must vanish at these points. Hence $\widetilde{\alpha}=\chi \alpha-U$ is holomorphic in $D_{1}$, and by the choice of $\chi$ we have that $\widetilde{\alpha}=\alpha$ on $X \cap D_{1}$ and $\widetilde{\alpha}(q, w)=0$, for all $w \in \mathbb{C}$.

We note that $\alpha(z, w)=\sum_{j=0}^{\infty}[F(z)]^{j} w^{j}$ and by the definition of $D_{1}$ we can write

$$
\widetilde{\alpha}(z, w)=\sum_{j=0}^{\infty} F_{j}(z) w^{j},
$$

where $F_{j}$ are holomorphic in $B^{n}$. Since for all $z \in B^{n}$ the analytic discs $\{(z, w): w \in \Delta\}$ are contained in $D_{1}$, it follows that

$$
\sum_{j=0}^{\infty} F_{j}(t u) w^{j}=\widetilde{\alpha}(t u, w)=\alpha(t u, w)=\sum_{j=0}^{\infty} t^{j} w^{j}
$$

and

$$
\sum_{j=0}^{\infty} F_{j}(q) w^{j}=\widetilde{\alpha}(q, w)=0,
$$

for all $t, w \in \Delta$. Hence for every $j \geq 1$ we have $F_{j}(t u)=t^{j}$, for all $t \in \Delta$, and $F_{j}(q)=0$. 
Finally, as the function $w \rightarrow \widetilde{\alpha}(z, w)$ is holomorphic in the disc $\{|w|<$ $\left.e^{-g_{n}(z, A) / \mu}\right\}$, it follows that $1 /\left(\limsup _{j \rightarrow \infty}\left|F_{j}(z)\right|^{1 / j}\right) \geq e^{-g_{n}(z, A) / \mu}$, for all $z \in B^{n}$. This proves conclusion (ii) of the theorem.

Remark. In view of the results of Lempert [Lm1] the last theorem can also be stated in the more general situation when $B^{n}$ is replaced by a strongly convex domain $\Omega$ with $C^{\infty}$ smooth boundary. Indeed, let us assume that $g_{\Omega}\left(z_{0}, A\right)=\mu g_{\Omega}\left(z_{0}, p\right)$ for some point $z_{0} \in \Omega \backslash\{p, q\}$, where $A=$ $\{(p, \mu),(q, \nu)\}, \mu \geq \nu$. By the results of Lempert there is a unique extremal disc $f: \Delta \rightarrow \Omega$ for the Kobayashi metric such that $f(0)=p$ and $f(\zeta)=z_{0}$, for some $\zeta \in(0,1)$. Along this disc we have $g_{\Omega}(f(t), p)=\log |t|$. Moreover, Lempert proved the existence of a holomorphic function $F: \Omega \rightarrow \Delta$ satisfying $F(f(t))=t$, for all $t \in \Delta$. In this setting, we can proceed as in the proof of the previous theorem to show the existence of a sequence of holomorphic functions $F_{j}: \Omega \rightarrow \mathbb{C}, j=1,2, \ldots$, which satisfy $F_{j}(q)=0, F_{j}(f(t))=t^{j}$, for all $t \in \Delta$, and $\lim \sup _{j \rightarrow \infty} \frac{1}{j} \log \left|F_{j}(z)\right| \leq g_{\Omega}(z, A) / \mu$, for all $z \in \Omega$.

Before we give the proof of Proposition 1, let us recall that a domain $\Omega$ in $\mathbb{C}^{n}$ is said to be taut if every sequence of holomorphic functions $f_{j}$ : $\Delta \rightarrow \Omega$ has a subsequence $\left\{f_{j_{k}}\right\}$ that either converges locally uniformly to a holomorphic function $f: \Delta \rightarrow \Omega$, or, for every compact sets $K \subset \Delta, L \subset \Omega$, one has $f_{j_{k}}(K) \subset \Omega \backslash L$ if $k$ is sufficiently large. In particular, if $\Omega$ is bounded and taut it follows from the classical Montel theorem that every sequence of holomorphic functions $f_{j}: \Delta \rightarrow \Omega$ has a subsequence which converges locally uniformly to a holomorphic function $f: \Delta \rightarrow \bar{\Omega}$ and either $f(\Delta) \subseteq \Omega$ or $f(\Delta) \subseteq \partial \Omega$.

Proof of Proposition 1. The assertions that the function $\delta_{\Omega}^{A}$ is negative with logarithmic poles in $A$ and that $g_{\Omega}(z, A) \leq \delta_{\Omega}^{A}(z)$ are obvious. The upper semicontinuity property holds for any bounded domain $\Omega$. Indeed, as the minimum of upper semicontinuous functions is upper semicontinuous, it is enough to check that the function $\delta_{\Omega}(\cdot, A)$ is upper semicontinuous. For $z \in \Omega \backslash\left\{p_{1}, \ldots, p_{k}\right\}$ and $\epsilon>0$ we fix a holomorphic function $f: \Delta \rightarrow \Omega$ such that $f(0)=z, f\left(s_{j}\right)=p_{j}$ for $j=1, \ldots, k$, and $\nu_{1} \log \left|s_{1}\right|+\cdots+\nu_{k} \log \left|s_{k}\right|<$ $\delta_{\Omega}(z, A)+\epsilon$. By shrinking $\Delta$ we may assume that $f$ is holomorphic in a neighborhood of $\bar{\Delta}$ and that $f(\bar{\Delta}) \subset \Omega$. Let $b: \mathbb{C} \rightarrow \mathbb{C}$ be the finite Blaschke product with zeros at $s_{1}, \ldots, s_{k}$, and let $z^{\prime} \in \Omega$. We define $\tilde{f}: \Delta \rightarrow \mathbb{C}^{n}$ by

$$
\tilde{f}(\zeta)=f(\zeta)+\frac{b(\zeta)}{b(0)}\left(z^{\prime}-z\right)
$$

Then $\tilde{f}(0)=z^{\prime}, \tilde{f}\left(s_{j}\right)=p_{j}$, and $\tilde{f}(\Delta) \subset \Omega$ provided that $z^{\prime}$ is sufficiently close to $z$; hence for such $z^{\prime}$ we have $\delta_{\Omega}\left(z^{\prime}, A\right)<\delta_{\Omega}(z, A)+\epsilon$.

We finally show that the function $\delta_{\Omega}^{A}$ is lower semicontinuous when $\Omega$ is taut. Let us assume for a contradiction that this does not hold at some point 
$z \in \Omega \backslash\left\{p_{1}, \ldots, p_{k}\right\}$. Then there is a sequence $\left\{z^{j}\right\}_{j} \subset \Omega$ and $\epsilon>0$ such that $z^{j} \rightarrow z$ and $\delta_{\Omega}^{A}\left(z^{j}\right)<\delta_{\Omega}^{A}(z)-\epsilon$. By the definition of $\delta_{\Omega}^{A}\left(z^{j}\right)$ we see that for each $j$ there is a holomorphic map $f_{j}: \Delta \rightarrow \Omega$ and a nonempty subset $S_{j}$ of $A$ with the following properties: $f_{j}(0)=z^{j}$, and for every $(p, \nu) \in S_{j}$ there is some $s_{j}(p) \in f_{j}^{-1}(p)$ such that

$$
\sum_{(p, \nu) \in S_{j}} \nu \log \left|s_{j}(p)\right|<\delta_{\Omega}^{A}(z)-\epsilon .
$$

Since $A$ is finite, it follows that $A$ has a nonempty subset $S$ such that $S_{j}=S$ for infinitely many $j$. So by passing to a subsequence we may assume that $S_{j}=S$ for all $j$. As $\Omega$ is bounded and taut it follows after passing to a subsequence that $\left\{f_{j}\right\}$ converges locally uniformly to a holomorphic map $f: \Delta \rightarrow \Omega$; moreover, we may assume (again by taking subsequences) that $s_{j}(p) \rightarrow s(p) \in \bar{\Delta}$, for every $(p, \nu) \in S$. By the above, we see that the set $S^{\prime}=\{(p, \nu) \in S: s(p) \in \Delta\}$ is clearly nonempty and

$$
\sum_{(p, \nu) \in S^{\prime}} \nu \log |s(p)| \leq \delta_{\Omega}^{A}(z)-\epsilon .
$$

Since $f(0)=z$ and $f(s(p))=p$ for $(p, \nu) \in S^{\prime}$, it follows that $\delta_{\Omega}\left(z, S^{\prime}\right) \leq$ $\delta_{\Omega}^{A}(z)-\epsilon$, which is in contradiction to the definition of $\delta_{\Omega}^{A}$.

Note. The extremal problem yielding the function $\delta_{n}(z, p, q)$ was also considered by E. Amar and P. J. Thomas in [AT1] (see in particular Section 5 and Section 6 of [AT1]). Their work is in connection with interpolating sequences in the unit ball for the space of bounded analytic functions. We also refer to [AT2] for related results regarding extremal analytic discs. I would like to thank Pascal Thomas for informing me about these results.

\section{References}

[AT1] E. Amar and P.J. Thomas, A notion of extremal analytic discs related to interpolation in the ball, Mathematische Annalen, 300 (1994), 419-433.

[AT2] - Continuity and convergence properties of extremal-interpolating disks, Publicacions Matematiques, 39 (1995), 335-347.

[BT] E. Bedford and B.A. Taylor, The Dirichlet problem for a complex Monge-Ampère equation, Inventiones Mathematicae, 37 (1976), 1-44.

[Br] H.J. Bremermann, On the conjecture of the equivalence of the plurisubharmonic functions and the Hartogs functions, Mathematische Annalen, 131 (1956), 76-86.

[C] D. Coman, Pluricomplex Green functions and the complex Monge-Ampère operator, Dissertation, The University of Michigan, 1997.

[D1] J.P. Demailly, Mesures de Monge-Ampère et mesures plurisousharmoniques, Mathematische Zeitschrift, 194 (1987), 519-564. 
[D2] _ Monge-Ampère operators, Lelong numbers and intersection theory, Complex analysis and geometry, Plenum, New York, (1993), 115-193.

[FS] J.E. Fornaess and N. Sibony, Oka's inequality for currents and applications, Mathematische Annalen, 301(3) (1995), 399-419.

[H1] L. Hörmander, An Introduction to Complex Analysis in Several Variables, 3rd rev. ed., North-Holland, Amsterdam New York, 1990.

[H2] Notions of Convexity, Birkhäuser, Boston-Basel-Berlin, 1994.

[K1] M. Klimek, Extremal plurisubharmonic functions and invariant pseudodistances, Bulletin de la Société Mathématique de France, 113 (1985), 231-240.

[K2] _ Pluripotential Theory, Clarendon Press, Oxford, 1991.

[L] P. Lelong, Fonction de Green pluricomplexe et lemmes de Schwarz dans les espaces de Banach, Journal de Mathématiques Pures et Appliquées; Neuvieme Serie, 68 (1989), 319-347.

[Lm1] L. Lempert, La métrique de Kobayashi et la représentation des domaines sur la boule, Bulletin de la Société Mathématique de France, 109 (1981), 427-474.

[Lm2] _ Solving the degenerate complex Monge-Ampère equation with one concentrated singularity, Mathematische Annalen, 263 (1983), 515-532.

[R] W. Rudin, Function Theory in the Unit Ball of $\mathbb{C}^{n}$, Springer-Verlag, New York Heidelberg Berlin, 1980.

[S] T.J. Suffridge, Common fixed points of commuting holomorphic maps of the hyperball, Michigan Mathematical Journal, 21 (1974), 309-314.

Received August 4, 1998 and revised May 24, 1999.

University of Notre DAME

Notre DAme, IN 46556-5683

E-mail address: Dan.F.Coman.2@nd.edu 